\title{
Dissipative solutions to compressible Navier-Stokes equations with general inflow-outflow data: existence, stability and weak strong uniqueness
}

\author{
Young-Sam Kwon * Antonin Novotny Vladyslav Satko \\ May 8, 2019 \\ Department of Mathematics, Dong-A University, \\ Busan 604-714, Republic of Korea \\ and \\ IMATH, EA 2134, Université du Sud Toulon-Var \\ BP 20132, 83957 La Garde, France \\ and \\ IMATH, EA 2134, Université du Sud Toulon-Var \\ BP 20132, 83957 La Garde, France
}

\begin{abstract}
So far existence of dissipative weak solutions for the compressible Navier-Stokes equations (i.e. weak solutions satisfying the relative energy inequality) is known only in the case of boundary conditions with non zero inflow/outflow (i.e., in particular, when the normal component of the velocity on the boundary of the flow domain is equal to zero). Most of physical applications (as flows in wind tunnels, pipes, reactors of jet engines) requires to consider non-zero inflow-outflow boundary condtions.

We prove existence of dissipative weak solutions to the compressible Navier-Stokes equations in barotropic regime (adiabatic coefficient $\gamma>3 / 2$, in three dimensions, $\gamma>1$ in two dimensions) with large velocity prescribed at the boundary and large density prescribed at the inflow boundary of a bounded piecewise regular Lipschitz domain, without any restriction neither on the shape of the inflow/outflow boundaries nor on the shape of the domain.

It is well known that the relative energy inequality has many applications, e.g., to investigation of incompressible or inviscid limits, to the dimension reduction of flows, to the error estimates of numerical schemes. In this paper we deal with one of its basic applications, namely weak-strong uniqueness principle.
\end{abstract}

*The work of Young-Sam Kwon was supported by Basic Science Research Program through the National Research Foundation of Korea(NRF) funded by the Ministry of Education(NRF- 2017R1D1A1B03030249) 
Keywords: Compressible Navier-Stokes system, inhomogeneous boundary conditions, weak solutions, dissipative solutions, relative energy inequality, weak-strong uniqueness, large inflow, large outflow

\section{Introduction}

We consider the system of equations governing the non steady motion of a compressible viscous fluid driven by general in/out flow boundary conditions on general bounded domains. The mass density $\varrho=\varrho(t, x)$ and the velocity $\mathbf{u}=\mathbf{u}(t, x),(t, x) \in I \times \Omega \equiv Q_{T}, I=(0, T)$ of the fluid satisfy the Navier-Stokes system,

$$
\begin{aligned}
\partial_{t} \varrho+\operatorname{div}_{x}(\varrho \mathbf{u}) & =0 \\
\partial_{t}(\varrho \mathbf{u})+\operatorname{div}_{x}(\varrho \mathbf{u} \otimes \mathbf{u})+\nabla_{x} p(\varrho) & =\operatorname{div}_{x} \mathbb{S}\left(\nabla_{x} \mathbf{u}\right),
\end{aligned}
$$

in $\Omega \subset R^{d}, d=2,3$, where the stress tensor is defined by

$$
\mathbb{S}\left(\nabla_{x} \mathbf{u}\right)=\mu\left(\nabla_{x} \mathbf{u}+\nabla_{x}^{t} \mathbf{u}\right)+\lambda \operatorname{div}_{x} \mathbf{u I}, \mu>0, \lambda \geq 0,
$$

and $p=p(\varrho)$ is the barotropic pressure.

The system is completed with initial conditions

$$
\varrho(0)=\varrho_{0}, \quad \varrho \mathbf{u}(0)=\varrho_{0} \mathbf{u}_{0}
$$

and boundary conditions

$$
\left.\mathbf{u}\right|_{\partial \Omega}=\mathbf{u}_{B},\left.\varrho\right|_{\Gamma_{\mathrm{in}}}=\varrho_{B},
$$

where

$$
\Gamma_{\text {in }}=\left\{x \in \partial \Omega \mid \mathbf{u}_{B} \cdot \mathbf{n}<0\right\}, \Gamma_{\text {out }}=\left\{x \in \partial \Omega \mid \mathbf{u}_{B} \cdot \mathbf{n}>0\right\} .
$$

In the above $\mathbf{n}$ is the outer normal to the boundary $\partial \Omega$.

The investigation and the better insight to the equations in this setting is important for many real world applications. In fact, this is a natural and basic abstract setting for flows in pipelines, wind tunnels, turbines to name a few concrete examples. Numerical modeling of fluid flow in the portions of cooling circuits of nuclear power stations, in the gas transporting industrial pipelines, in the reactors of jet engines and in many other situations, requires this or similar boundary value setting rather than academic no-slip, Navier or periodic boundary conditions.

Existence of (renormalized bounded energy) weak solutions for system (1.1]1.5) is well known -for the pressure $p(\varrho)$ behaving as $\varrho^{\gamma}$ at infinity with $\gamma>d / 2$ - in the "simple" case of zero inflow and outflow boundary data with no-slip or slip (Navier) boundary conditions (when, in particular, $\left.\mathbf{u} \cdot \mathbf{n}\right|_{\partial \Omega}=0$ ) since the end of the last century/beginning of this century, cf. [15], Feireisl[11] and monographs by Lions [22], Feireisl [10] and [24] (see also an alternative approach by Bresch, Jabin [1] working for $\gamma \geq 9 / 5(d=3)$ with possibly non monotone pressure). In this situation, it is also known, that any bounded energy weak solution is dissipative, meaning that it obeys the so called relative energy inequality and consequently 
satisfies, in particular, the weak strong uniqueness principle, see [13], [16] (and the seminal paper of Dafermos [6] for the general introduction of relative energy (entropy) method in the fluid mechanics).

Existence of (renormalized bounded energy) weak solutions for system (1.1 1.5) with large boundary data exhibits many additional difficulties. It was investigated recently in Novo [23, Girinon [21] with several geometrical restrictions and in [3], 5] in full generality. If the initial data and the boundary are smooth, the same problem admits local in time strong solutions which become global if the initial data are sufficiently small, see Valli, Zajaczkowski [25]. A general question arises whether strong solutions are unique in the class of weak solutions, at least on the lifespan of the former. In contrast to the situation with no inflow/outflow, in this general setting, it is not known, whether the class of renormalized bounded energy weak solution coincides with the class of dissipative solutions, i.e., whether the weak strong uniqueness is true. This question remains apparently an interesting open problem.

We are, however, able to construct a subclass of weak solutions, called dissipative weak solutions, that obey, in particular, the weak strong uniqueness principle. The theorem (and proof) of the existence of (renormalized bounded energy) dissipative weak solutions is the first main goal of the present paper.

Except its important application potential, there are notably two features that distinguish our result from the similar result known for the no-slip (or Navier) boundary conditions:

1. The test density in the relative energy inequality cannot be taken arbitrarily but must obey the continuity equation with transporting velocity which is an arbitrary vector field satisfying the boundary conditions of the problem, and which is at the same time the test velocity in the relative energy inequality.

2. Presuure $p(\varrho)$ is only $L^{1}$-integrable near the boundary. In absence of higher integrability, one must show that it is "equi-integrable" near the boundary. This result is formulated in Lemma 6.1 and it is of independent interest.

As in the case of zero boundary conditions, this result (in particular, the relative energy inequality) opens the way to many applications in geometrical setting with non zero inflow/outflow that are really interesting from the engineering and physical points of view both on the theoretical level (as e.g. rigorous investigation of model reduction through singular distinguished limits that plays crucial role in derivation of simplified models in the physics of atmosphere, cf. Klein et al. [19], in the spirit of [14, 2nd. edition, Chapter 9]) and on the level of numerical analysis (as e.g. derivation of rigorous unconditional error estimates in the spirit of [17, [18]), which were, so far, out of reach of the analysis.

In this paper, we develop one of them, namely, we prove stability of strong solutions in the class of dissipative weak solutions and the weak-strong uniqueness principle for the dissipative weak solutions. This is the second main result of the present paper.

The paper is organized as follows. In Section 2 we expose the definition of dissipative weak solutions and state the main theorems (Theorem 2.2 about the existence of weak dissipative solutions and Theorem 2.4 about the stability of strong solutions in the class of weak solutions and the weak strong uniqueness). The construction of solutions is explained in Section 3 which provides in Lemma 3.1 existence of generalized solutions to the approximate system. This lemma is proved in Section 4 . This is the real starting point of the topic. A relative energy inequality for the approximate problem is derived 
in Section 5, see Lemma 5.1. It is the starting point of the proof of Theorem 2.2 which is performed in Sections 6 and 7 . Section 8 is devoted to the proof of Theorem 2.4. Finally, in Section 9 we show that the results of Theorems 2.2 and 2.4 can be extended to the piecewise regular Lipschitz domains and to certain nonmonotone pressure laws.

Throughout the paper, we use the standard notation for Sobolev and Bochner spaces, see, e.g., the book of Evans [9].

\section{Main results}

In order to avoid additional technicalities, we suppose that the domain and boundary data satisfy

$$
\Omega \text { a bounded domain, } \partial \Omega \in C^{2}, \mathbf{u}_{B} \in C^{2}\left(\partial \Omega ; R^{d}\right), 0<\underline{\varrho}_{B} \leq \varrho_{B} \leq \bar{\varrho}_{B}, \varrho_{B} \in C\left(\bar{\Gamma}_{\mathrm{in}}\right) \text {. }
$$

The results however hold also for piecewise $C^{2}$ Lipschitz domains. This extension will be discussed in the last section.

Throughout the paper, we consider strictly increasing pressure satisfying at least

$$
p \in C[0, \infty) \cap C^{1}(0, \infty), p(0)=0, p^{\prime}(\varrho)>0 .
$$

All results of this paper can be however extended to certain non-monotone pressure laws (with nonmonotonicity at least at a compact portion of the interval $[0, \infty)$ ). We shall comment about this issue in the last section.

For further convenience, it will be useful to introduce the Helmholtz function

$$
H(\varrho)=\varrho \int_{1}^{\varrho} \frac{p(z)}{z^{2}} \mathrm{~d} z
$$

and the relative energy function

$$
E(\varrho \mid r)=H(\varrho)-H^{\prime}(r)(\varrho-r)-H(r)
$$

One can verify by the direct calculation that

$$
\varrho H^{\prime}(\varrho)-H(\varrho)=p(\varrho) \text { and consequently } H^{\prime \prime}(\varrho)=\frac{p^{\prime}(\varrho)}{\varrho} \text {. }
$$

We begin with the definition of dissipative weak solutions to system (1.1 1.5). In this definition, $\Omega$ is a bounded Lipschitz domain, $p \in C[0, \infty) \cap C^{1}(0, \infty)$ and $\mathbf{u}_{B} \in W^{1, \infty}(\partial \Omega), \varrho_{B} \in L^{\infty}(\partial \Omega)$.

Definition 2.1 [Dissipative weak solutions to system (1.1 1.5)] We say that $(\varrho, \mathbf{u})$ is a dissipative weak solution of problem (1.1-1.5) if: 
1. There exists a Lipschitz extension $\mathbf{u}_{\infty} \in W^{1, \infty}\left(\Omega ; \mathbb{R}^{d}\right)$ of $\mathbf{u}_{B}$ whose divergence is non negative in a certain interior neighborhood of $\partial \Omega$, i.e.

$$
\operatorname{div} \mathbf{u}_{\infty} \geq 0 \text { a.e. in } \hat{U}_{h}^{-} \equiv\{x \in \Omega \mid \operatorname{dist}(x, \partial \Omega)<h\}, h>0
$$

and

$$
\begin{aligned}
& \varrho \in L^{\infty}\left(0, T ; L^{\gamma}(\Omega)\right) \text { with some } \gamma>1,0 \leq \varrho \text { a.e. in }(0, T) \times \Omega \\
& p(\varrho) \in L^{1}\left(Q_{T}\right), \mathbf{v}:=\mathbf{u}-\mathbf{u}_{\infty} \in L^{2}\left(0, T ; W_{0}^{1,2}\left(\Omega ; R^{d}\right)\right) .
\end{aligned}
$$

2. Function $\varrho \in C_{\text {weak }}\left([0, T], L^{\gamma}(\Omega)\right)_{1}^{1}$ and the integral identity

$$
\begin{aligned}
& \int_{\Omega} \varrho(\tau, \cdot) \varphi(\tau, \cdot) \mathrm{d} x-\int_{\Omega} \varrho_{0}(\cdot) \varphi(0, \cdot) \mathrm{d} x \\
& =\int_{0}^{\tau} \int_{\Omega}\left(\varrho \partial_{t} \varphi+\varrho \mathbf{u} \cdot \nabla_{x} \varphi\right) \mathrm{d} x \mathrm{~d} t-\int_{0}^{\tau} \int_{\Gamma_{\mathrm{in}}} \varrho_{B} \mathbf{u}_{B} \cdot \mathbf{n} \varphi \mathrm{d} S_{x} \mathrm{~d} t
\end{aligned}
$$

holds for any $\tau \in[0, T]$ and $\varphi \in C_{c}^{1}\left([0, T] \times\left(\Omega \cup \Gamma_{\text {in }}\right)\right)$.

3. Function $\varrho \mathbf{u} \in C_{\text {weak }}\left([0, T], L^{\frac{2 \gamma}{\gamma+1}}\left(\Omega ; R^{d}\right)\right)$, and the integral identity

$$
\begin{aligned}
& \int_{\Omega} \varrho \mathbf{v}(\tau, \cdot) \cdot \boldsymbol{\varphi}(\tau, \cdot) \mathrm{d} x-\int_{\Omega} \varrho_{0} \mathbf{v}_{0}(\cdot) \boldsymbol{\varphi}(0, \cdot) \mathrm{d} x \\
& =\int_{0}^{\tau} \int_{\Omega}\left(\varrho \mathbf{v} \cdot \partial_{t} \varphi+\varrho \mathbf{u} \cdot \nabla_{x} \boldsymbol{\varphi} \cdot \mathbf{u}-\varrho \mathbf{u} \cdot \nabla_{x}\left(\mathbf{u}_{\infty} \cdot \boldsymbol{\varphi}\right)+p(\varrho) \operatorname{div}_{x} \boldsymbol{\varphi}-\mathbb{S}\left(\nabla_{x} \mathbf{u}\right): \nabla_{x} \boldsymbol{\varphi}\right) \mathrm{d} x \mathrm{~d} t
\end{aligned}
$$

holds for any $\tau \in[0, T]$ and any $\varphi \in C_{c}^{1}\left([0, T] \times \Omega ; R^{d}\right)$.

4. Function $\varrho|\mathbf{u}|^{2} \in L^{\infty}\left(I ; L^{1}(\Omega)\right)$ and the so called relative energy inequality

$$
\begin{aligned}
& \int_{\Omega}\left(\frac{1}{2} \varrho|\mathbf{v}-\mathbf{V}|^{2}+E(\varrho \mid r)\right)(\tau) \mathrm{d} x+\int_{0}^{\tau} \int_{\Omega} \mathbb{S}\left(\nabla_{x} \mathbf{u}\right): \nabla_{x}(\mathbf{v}-\mathbf{V}) \mathrm{d} x \mathrm{~d} t \\
& \leq \int_{\Omega}\left(\frac{1}{2} \varrho_{0}\left|\mathbf{u}_{0}-\mathbf{U}(0, \cdot)\right|^{2}+E\left(\varrho_{0} \mid r(0, \cdot)\right)\right) \mathrm{d} x \\
& \quad+\int_{0}^{\tau} \int_{\Gamma_{\mathrm{in}}}\left(H(r)-r H^{\prime}(r)-H\left(\varrho_{B}\right)+\varrho_{B} H^{\prime}(r)\right) \mathbf{u}_{B} \cdot \mathbf{n} \mathrm{d} S \mathrm{~d} t \\
& \quad+\int_{0}^{\tau} \int_{\Omega}\left(\varrho(\mathbf{V}-\mathbf{v}) \cdot \partial_{t} \mathbf{V}+\varrho \mathbf{u} \cdot \nabla_{x} \mathbf{U} \cdot(\mathbf{V}-\mathbf{v})\right) \mathrm{d} x \mathrm{~d} t \\
& \quad+\int_{0}^{\tau} \int_{\Omega}\left(p(r)-p^{\prime}(r)(r-\varrho)-p(\varrho)\right) \operatorname{div} \mathbf{U} \mathrm{d} x \mathrm{~d} t
\end{aligned}
$$

\footnotetext{
${ }^{1}$ We say that $f \in C_{\text {weak }}\left([0, T], L^{p}(\Omega)\right)$ iff $f:[0, T] \rightarrow L^{p}(\Omega)$ is defined everywhere on $[0, T], f \in L^{\infty}\left(0, T ; L^{p}(\Omega)\right)$ and the map $t \mapsto \int_{\Omega} f \varphi(t, \cdot) \mathrm{d} x \in C[0, T]$ for all $\varphi \in L^{p^{\prime}}(\Omega)$.
} 


$$
+\int_{0}^{\tau} \int_{\Omega}\left(-p^{\prime}(r) \mathbf{v} \cdot \nabla_{x} r-p(r) \operatorname{div} \mathbf{V}+\frac{r-\varrho}{r} p^{\prime}(r)(\mathbf{v}-\mathbf{V}) \cdot \nabla_{x} r\right) \mathrm{d} x \mathrm{~d} t
$$

holds with a.e. $\tau \in I$ and with any test functions $(r, \mathbf{U})$,

$$
\mathbf{U}=C^{1}(\bar{Q}),\left.\mathbf{U}\right|_{\bar{I} \times \partial \Omega}=\mathbf{u}_{B}, \mathbf{V}=: \mathbf{U}-\mathbf{u}_{\infty}
$$

and

$$
0<r \in C^{1}\left(\overline{Q_{T}}\right) \text { satisfying } \partial_{t} r+\operatorname{div}(r \mathbf{U})=0 .
$$

Remark 2.1. 1. A Lipschitz extension $\mathbf{u}_{\infty}$ of $\mathbf{u}_{B}$ verifying (2.6) always exists. Indeed, according to [21, Lemma 3.3]), for any $\mathbf{V} \in W^{1, \infty}\left(\partial \Omega ; \mathbb{R}^{d}\right)$ (where $\Omega \subset R^{d}$ is a bounded Lipschitz domain) there is $h>0$ and a vector field

$$
\mathbf{V}_{\infty} \in W^{1, \infty}(\Omega), \quad \operatorname{div} \mathbf{V}_{\infty} \geq 0 \text { a.e. } \operatorname{in} \hat{U}_{h}^{-}
$$

verifying $\left.\mathbf{V}_{\infty}\right|_{\partial \Omega}=\mathbf{V}$.

2. If $\Omega$ is bounded domain of class $C^{2}$ and $\int_{\partial \Omega} \mathbf{u}_{B} \cdot \mathbf{n d} S=0$ then $\mathbf{u}_{\infty}$ can be chosen in such a way that

$$
\operatorname{div} \mathbf{u}_{\infty}=0 \text { in } \Omega
$$

see Galdi [20, Theorem IV.6.1].

3. If $\partial \Omega$ is in class $C^{2}$, given $\mathbf{U}$ in class (2.11), the Cauchy-Lipschitz theory guarantees that equation (2.12) admits infinitely many solutions $r$ in class (2.12) according to the free choice of the initial condition. They can be constructed by the method of characteristics e.g. as follows: One extends U to $\tilde{\mathbf{U}} \in C_{c}^{1}\left(R \times R^{d}\right)$ and finds its flow $X(\cdot ; \cdot)$ (by definition $X(\cdot, x)$ is the (unique) solution of the ODE $\left.y^{\prime}(t)=\tilde{\mathbf{U}}(t, y), y(0)=x\right)$. We know that $X \in C^{1}\left(R \times R^{d}\right)$, for all $t \in R, X(t ; \cdot)$ is a bijection from $R^{d}$ to $R^{d}$ and $X(t+s, x)=X(t ; X(s ; x))$, cf. [2, Theorem 5.13]. Consequently, for any given $\mathfrak{R}_{0} \in C^{1}\left(R^{d}\right)$ function $\mathfrak{R}(t, x)=\mathfrak{R}_{0}(X(-t ; x)) \exp \left(-\int_{0}^{t} \operatorname{div} \tilde{\mathbf{U}}(s, X(s-t ; x)) \mathrm{d} s\right.$ solves the continuity equation $\partial_{t} \mathfrak{R}+\operatorname{div}(\mathfrak{R} \tilde{\mathbf{U}})=0$ in $R \times R^{d}$. Now, it is enough to take $r=\left.\mathfrak{R}\right|_{[0, T] \times \bar{\Omega}}$.

4. Equation (2.8) implies the total mass inequality

$$
\int_{\Omega} \varrho(\tau) \mathrm{d} x \leq \int_{\Omega} \varrho_{0} \mathrm{~d} x-\int_{0}^{\tau} \int_{\Gamma_{\mathrm{in}}} \varrho_{B} \mathbf{u}_{B} \cdot \mathbf{n} \mathrm{d} S_{x} \mathrm{~d} t
$$

for all $\tau \in[0, T]$. To see it, it is enough to take for the test functions in (2.8) a convenient sequence $\varphi=\varphi_{\varepsilon}, \varepsilon>0$ as, e.g.,

$$
\varphi_{\varepsilon}(x)=\left\{\begin{array}{l}
1 \text { if } \operatorname{dist}\left(x, \Gamma_{\text {out }}\right)>\varepsilon \\
\frac{1}{\varepsilon} \operatorname{dist}\left(x, \Gamma_{\text {out }}\right) \text { if } \operatorname{dist}\left(x, \Gamma_{\text {out }}\right) \leq \varepsilon
\end{array}\right\}
$$

and send $\varepsilon \rightarrow 0$. 
5. Regularity of the test functions $r, \mathbf{U}$ in (2.11), (2.12), can be weaken, in particular, up to $r$, U continuous functions on $\overline{Q_{T}}$ and $\partial_{t}(r, \mathbf{U}), \nabla_{x}(r, \mathbf{U}) \in L^{2}(I ; C(\bar{\Omega}))$.

6. One can define bounded energy weak solutions requiring satisfaction of Items 1.-3. of Definition 2.1, and energy inequality which reads,

$$
\begin{gathered}
\int_{\Omega}\left(\frac{1}{2} \varrho|\mathbf{v}|^{2}+H(\varrho)\right)(\tau) \mathrm{d} x+\int_{0}^{\tau} \int_{\Omega} \mathbb{S}\left(\nabla_{x} \mathbf{v}\right): \nabla_{x} \mathbf{v} \mathrm{d} x \mathrm{~d} t \\
\leq \int_{\Omega}\left(\frac{1}{2} \varrho_{0}\left|\mathbf{v}_{0}\right|^{2}+H\left(\varrho_{0}\right)\right) \mathrm{d} x-\int_{0}^{\tau} \int_{\Gamma_{\mathrm{in}}} H\left(\varrho_{B}\right) \mathbf{u}_{B} \cdot \mathbf{n} \mathrm{d} S_{x} \mathrm{~d} t \\
+\int_{0}^{\tau} \int_{\Omega}\left(-p(\varrho) \operatorname{div} \mathbf{u}_{\infty}-\mathbb{S}\left(\nabla_{x} \mathbf{u}_{\infty}\right): \nabla_{x} \mathbf{v}-\varrho \mathbf{u} \cdot \nabla_{x} \mathbf{u}_{\infty} \cdot \mathbf{v}\right) \mathrm{d} x \mathrm{~d} t
\end{gathered}
$$

see [3, Definition 2.1].

We shall construct the dissipative weak solutions in such a way that they are also the bounded energy weak solutions, i.e., they satisfy, in addition to all items in Definition 2.1, also the energy inequality (2.16).

A natural question arises whether any dissipative weak solution is a bounded energy weak solution, i.e. if it satisfies also (2.16). We recall that in the case of zero inflow/outflow boundary conditions, the answer is "yes" and its proof takes one line, see [13. In the case of non zero inflow/outflow boundary conditions the answer is "yes", at least provided the function $p(z) / z^{2}$ is integrable near 0 . In this case, one can suppose without the loss of generality that $H^{\prime}$ is a positive function. The process of deducing from the relative energy inequality (2.10) the energy inequality (2.16) goes as follows: We take 1) in (2.8) the test function $\left.\varphi=-\frac{1}{2}|\mathbf{V}|^{2}, 2\right)$ in (2.9) the test function $\varphi=\mathbf{V}$ and finally 3) in (2.8) the test function $\varphi=H^{\prime}(r) \varphi_{\varepsilon}$ (cf. (2.15)). Adding the results of all three above steps to (2.10), we obtain, after a long calculation (which uses identities (2.12) and (2.5)) and after sending $\varepsilon \rightarrow 0$, the energy inequality (2.16). The crucial point in this process is the treatment in the limit of terms $\int_{0}^{\tau} \int_{\Omega} H^{\prime}(r)\left(\mathbf{u}-\mathbf{u}_{\infty}\right) \cdot \nabla_{x} \varphi_{\varepsilon} \mathrm{d} x \mathrm{~d} t$ and $\left.\int_{0}^{\tau} \int_{\Omega} H^{\prime}(r) \mathbf{u}_{\infty}\right) \cdot \nabla_{x} \varphi_{\varepsilon} \mathrm{d} x \mathrm{~d} t$ in step 3$)$. In fact, $\lim _{\varepsilon \rightarrow 0}$ of the first one is equal to 0 by virtue of the Hardy inequality and $\lim _{\sup } \operatorname{su}_{\varepsilon \rightarrow 0}$ of the second one is non positive provided $H^{\prime}(r) \geq 0\left(\right.$ since $\nabla_{x} \operatorname{dist}\left(x, \Gamma_{\text {out }}\right) \rightarrow-\mathbf{n}\left(x_{0}\right)$ as $\left.x \rightarrow x_{0} \in \Gamma_{\text {out }}\right)$.

Definition 2.2 We say that the couple $(\varrho, \mathbf{u}) \in C_{\text {weak }}\left(\bar{I} ; L^{p}(\Omega)\right) \times L^{2}\left(0, T ; W^{1,2}\left(\Omega, \mathbb{R}^{d}\right)\right), p>1$ is a renormalized solution of the continuity equation if $b(\varrho) \in C_{\text {weak }}\left([0, T] ; L^{1}(\Omega)\right.$ ) (not relabeled in time) and if it satisfies in addition to the continuity equation (2.8) also equation

$$
\begin{aligned}
& \int_{\Omega}(b(\varrho) \varphi)(\tau) \mathrm{d} x-\int_{\Omega} b\left(\varrho_{0}\right) \varphi(0) \mathrm{d} x \\
& =\int_{0}^{\tau} \int_{\Omega}\left(b(\varrho) \partial_{t} \varphi b(\varrho) \mathbf{u} \cdot \nabla_{x} \varphi-\varphi\left(b^{\prime}(\varrho) \varrho-b(\varrho)\right) \operatorname{div}_{x} \mathbf{u}\right) \mathrm{d} x \mathrm{~d} t-\int_{0}^{\tau} \int_{\Gamma_{\mathrm{in}}} b\left(\varrho_{B}\right) \mathbf{u}_{B} \cdot \mathbf{n} \varphi \mathrm{d} S_{x} \mathrm{~d} t
\end{aligned}
$$

for any $\varphi \in C_{c}^{1}\left([0, T] \times\left(\Omega \cup \Gamma_{\text {in }}\right)\right)$, and any continuously differentiable $b$ with $b^{\prime}$ having a compact support in $[0, \infty)$. 
A (dissipative) weak solution to problem (1.1 1.5) satisfying in addition renormalized continuity equation (2.17) is called a renormalized (dissipative) weak solution.

Our first main result is the following theorem.

Theorem 2.2. [Existence of dissipative weak solutions] Let $\Omega \subset R^{d}, d=2,3$ be a bounded domain of class $C^{2}$. Let the boundary data $\mathbf{u}_{B}, \varrho_{B}$ satisfy (2.1). Assume that the pressure satisfies hypotheses (2.2) and

$$
p^{\prime}(\varrho) \geq a_{1} \varrho^{\gamma-1}-b, p(\varrho) \leq a_{2} \varrho^{\gamma}+b, \gamma>d / 2, a_{1}, a_{2}>0, b \geq 0 .
$$

Suppose finally that the initial data have the finite energy and the finite mass,

$$
\int_{\Omega}\left(\frac{1}{2} \varrho_{0}\left|\mathbf{u}_{0}\right|^{2}+H\left(\varrho_{0}\right)\right) \mathrm{d} x<\infty, \quad 0 \leq \varrho_{0}, \int_{\Omega} \varrho_{0} \mathrm{~d} x>0 .
$$

Then for any Lipschitz extension $\mathbf{u}_{\infty}$ of $\mathbf{u}_{B}$ verifying (2.6) problem (1.1 1.5) possesses at least one renormalized dissipative weak solution $(\varrho, \mathbf{u})$ which satisfies the energy inequality (2.16).

Remark 2.3. 1. Theorem 2.2 still holds provided one considers in the momentum equation at its right hand side the term @f, $\mathbf{f} \in L^{\infty}\left(Q_{T}\right)$, corresponding to the action of large external forces. The necessary changes in the weak formulation and in the relative energy inequality in order to accommodate the presence of this term are left to the reader.

2. Conditions on the regularity $p, \varrho_{B}$ and $\mathbf{u}_{B}$ in Theorem 2.2 could be slightly weakened, up to $p$ continuous on $[0, \infty)$, locally Lipschitz on $[0, \infty), \varrho_{B} \in L^{\infty}(\partial \Omega), \mathbf{u}_{B} \in W^{1, \infty}(\partial \Omega)$, at expense of some additional technical difficulties.

3. We shall perform the proof in all details in the case $d=3$ assuming tacitly that both $\Gamma_{\text {in }}$ and $\Gamma_{\text {out }}$ have non zero $(d-1)$-Hausdorff measure. Other cases, namely the case $d=2$ is left to the reader as an exercise.

Our second main result is the following.

Theorem 2.4. [Stability and Weak-strong uniqueness principle] Let $\Omega$ be a bounded Lipschitz domain. Suppose that the pressure is in addition to (2.2), twice continuously differentiable on $(0, \infty)$ and obeys

$$
c(\varrho+H(\varrho)) \geq p(\varrho) \text { for all } \varrho \geq \bar{R}
$$

where $\bar{R}$, c are some positive constants. Assume that the initial data $\varrho_{0}, \mathbf{u}_{0}$ verify condition (2.19) and boundary data satisfy condition (2.1). Let $\mathbf{u}_{\infty}$ be an Lipschitz extension of $\mathbf{u}_{B}$ satisfying conditions (2.13).

Let $(\varrho, \mathbf{u})$ be a dissipative weak solution to the Navier-Stokes equations (1.1]1.5) associated to the extension $\mathbf{u}_{\infty}$ of $\mathbf{u}_{B}$. Let $(r, \mathbf{U})$ that belongs to the class

$$
0<\underline{r} \leq r \leq \bar{r}<\infty ; \quad \mathbf{U} \in L^{\infty}((0, T) \times \Omega)
$$




$$
\partial_{t} r, \partial_{t} \mathbf{U}, \nabla_{x} r, \nabla_{x} \mathbf{U} \in L^{2}(0, T ; C(\bar{\Omega}))^{2}
$$

be a strong solution of the same equations with initial data $\left(r_{0}, \mathbf{U}_{0}\right)$ and boundary data $\left(\left.r\right|_{\Gamma_{\mathrm{in}}}=r_{B},\left.\mathbf{U}\right|_{\partial \Omega}=\right.$ $\left.\mathbf{u}_{B}\right)$.

1. Then there exists

$$
c=c\left(\mu, T,|\Omega|, \operatorname{diam} \Omega, \mu, \underline{\mathfrak{r}}, \overline{\mathfrak{r}},\left|p, p^{\prime}, H^{\prime}\right|_{C^{1}(\underline{\mathfrak{r}} / 2,2 \overline{\mathfrak{r}})},\left\|\partial_{t} \mathbf{V}, \nabla \mathbf{V}, \nabla r\right\|_{L^{2}\left(0, T ; L^{\infty}\left(\Omega ; R^{15}\right)\right)}\right)>0
$$

(where $\left.\underline{\mathfrak{r}}=\min \left\{\underline{r}, \underline{\varrho}_{B}\right\}, \overline{\mathfrak{r}}=\max \left\{\bar{r}, \bar{\varrho}_{B}\right\}\right)$, such that

$$
\mathcal{E}(\varrho, \mathbf{v} \mid r, \mathbf{V})(\tau) \leq c\left(\mathcal{E}\left(\varrho_{0}, \mathbf{v}_{0} \mid r_{0}, \mathbf{U}_{0}\right)+\left\|\varrho_{B}-r_{B}\right\|_{L^{1}\left(\Gamma_{\text {in }}\right)}\right)
$$

for a.e. $\tau \in(0, T)$, where $\mathbf{v}=\mathbf{u}-\mathbf{u}_{\infty}, \mathbf{V}=\mathbf{U}-\mathbf{u}_{\infty}, \mathbf{v}_{0}=\mathbf{u}_{0}-\mathbf{u}_{\infty}, \mathbf{V}_{0}=\mathbf{U}_{0}-\mathbf{u}_{\infty}$. In the above, we have denoted

$$
\mathcal{E}(\varrho, \mathbf{v} \mid r, \mathbf{V}):=\int_{\Omega}\left(\frac{1}{2} \varrho|\mathbf{v}-\mathbf{V}|^{2}+E(\varrho \mid r)\right) \mathrm{d} x
$$

the relative energy functional.

2. In particular, if $\left(\varrho_{0}, \mathbf{u}_{0}\right)=\left(r_{0}, \mathbf{U}_{0}\right)$, and $r_{B}=\varrho_{B}$ then

$$
\varrho=r, \mathbf{u}=\mathbf{U} \text { in }[0, T] \times \Omega .
$$

Remark 2.5. 1. The (dissipative) weak-strong uniqueness holds for pressure functions fairly beyond the conditions (2.18) guaranteeing existence of weak solutions. An example of an admissible class of pressure functions is the class $p \in C[0, \infty) \cap C^{2}(0, \infty), p(0)=0, p^{\prime}(\varrho)>0$ and

$$
0<\frac{1}{p_{\infty}} \leq \liminf _{\varrho \rightarrow \infty} \frac{p(\varrho)}{\varrho^{\gamma}} \leq \limsup _{\varrho \rightarrow \infty} \frac{p(\varrho)}{\varrho^{\gamma}} \leq p_{\infty}<\infty, \gamma \geq 1
$$

Indeed, one can easy verify, that condition (2.24) yields condition (2.20) from Theorem 2.4, In particular, condition (2.18) implies (2.24), whence also (2.20). Consequently, dissipative weak solutions constructed in Theorem 2.2 satisfy the weak-strong uniqueness principle.

2. Existence of strong solutions at least on a short time interval is well known. Here we report the following existence result of Valli and Zajaczkowski [25, Theorem 2.5].

Lemma 2.6. Let $D$ be a positive constant, $\Omega$ a bounded domain of class $C^{3}$ and $p \in C^{2}((0, \infty))$. Let

$$
\mathbf{u}_{\infty} \in W^{3,2}(\Omega), \mathbf{u}_{B}=\left.\mathbf{u}_{\infty}\right|_{\partial \Omega}, \varrho_{B} \in W^{2,2}\left(\Gamma_{\mathrm{in}}\right)
$$

${ }^{2}$ The requested regularity of some of this derivatives can be slightly weaken by a more detailed (elementary) analysis as in [13]. 
where the inflow boundary $\Gamma_{\text {in }}$ is define in (1.5). Assume further that

$$
\begin{gathered}
\mathbf{u}_{0} \in W^{3,2}\left(\Omega ; R^{3}\right),\left.\mathbf{u}_{0}\right|_{\partial \Omega}=\mathbf{u}_{B} \\
\varrho_{0} \in W^{2,2}(\Omega), \inf _{\Omega} \varrho_{0}>0,\left.\varrho_{0}\right|_{\Gamma_{\text {in }}}=\varrho_{B},
\end{gathered}
$$

where

$$
\begin{gathered}
\left.\operatorname{div}\left(\varrho_{0} \mathbf{u}_{0}\right)\right|_{\Gamma_{\text {in }}}=0 \\
\left.\frac{1}{\varrho_{0}}\left(-\nabla_{x} p\left(\varrho_{0}\right)+\operatorname{div} \mathbb{S}\left(\nabla_{x} \mathbf{u}_{0}\right)-\varrho_{0} \mathbf{u}_{0} \cdot \nabla_{x} \mathbf{u}_{0}\right)\right|_{\partial \Omega}=0 .
\end{gathered}
$$

Then there exists $T=T_{0}(D)$ such that if

$$
\left\|\varrho_{0}\right\|_{W^{2,2}(\Omega)}+\left\|\mathbf{u}_{0}\right\|_{W^{3,2}(\Omega)}+1 / \inf _{\Omega} \varrho_{0}+\left\|\mathbf{u}_{\infty}\right\|_{W^{3,2}(\Omega)}+\left\|\varrho_{B}\right\|_{W^{2,2}\left(\Gamma_{\mathrm{in}}\right)} \leq D
$$

then the problem (1.1 1.5) admits a unique strong solution (in the sense a.e. in $(0, T) \times \Omega$ ) in the class

$$
\begin{gathered}
\varrho \in C\left([0, T) ; W^{2,2}(\Omega)\right), \mathbf{u}-\mathbf{u}_{\infty} \in C\left([0, T) ; W^{3,2} \cap W_{0}^{1,2}\left(\Omega ; R^{3}\right)\right) \cap L^{2}\left(0, T ; W^{4,2}\left(\Omega ; R^{3}\right)\right), \\
\partial_{t} \varrho \in C\left([0, T) ; W^{1,2}(\Omega)\right), \partial_{t} \mathbf{u} \in L^{2}\left([0, T) ; W^{2,2}\left(\Omega ; R^{3}\right)\right) .
\end{gathered}
$$

In particular,

$$
0<\underline{r} \equiv \inf _{(t, x) \in(0, T) \times \Omega} \varrho(t, x) \leq \sup _{(t, x) \in(0, T) \times \Omega} \varrho(t, x) \equiv \bar{r}<\infty
$$

\section{Approximate problem}

Our goal is to construct a dissipative weak solution, the existence of which is claimed in Theorem 2.2 . We shall use the approximating procedure suggests in [3]. We add to the pressure an artificial pressure (small parameter $\delta>0$ ), regularize the continuity equation by adding an artificial viscosity term to equation (1.1) endowing at the same time the momentum equation by a compensation term in order to keep the energy inequality - this is so far standard - and add to the momentum equation a monotone dissipation (the latter adjustment is mostly technical) - all these three ingredients parametrized by a small parameter $\varepsilon>0$. Moreover, the regularized continuity equation is completed with boundary conditions through a boundary operator which gives in the limit $\varepsilon \rightarrow 0$ the general inflow/outflow conditions.

The approximating system of equations reads:

$$
\begin{gathered}
\partial_{t} \varrho-\varepsilon \Delta_{x} \varrho+\operatorname{div}_{x}(\varrho \mathbf{u})=0, \\
\varrho(0, x)=\varrho_{0}(x),\left.\left(-\varepsilon \nabla_{x} \varrho \cdot \mathbf{n}+\varrho v\right)\right|_{I \times \partial \Omega}=g,
\end{gathered}
$$


where

$$
\begin{gathered}
\left\{\begin{array}{c}
\mathbf{u}_{B} \cdot \mathbf{n} \text { on } \Gamma_{\text {in }}, \\
0 \text { on } \partial \Omega \backslash \Gamma_{\text {in }}
\end{array}\right\} \equiv v, \quad \varrho_{B} v \equiv g . \\
\partial_{t}(\varrho \mathbf{u})+\operatorname{div}_{x}(\varrho \mathbf{u} \otimes \mathbf{u})+\nabla_{x} p_{\delta}(\varrho)=\operatorname{div}_{x} \mathbb{S}\left(\nabla_{x} \mathbf{u}\right)-\varepsilon \nabla_{x} \varrho \cdot \nabla_{x} \mathbf{u}+\varepsilon \operatorname{div}\left(\left|\nabla_{x}\left(\mathbf{u}-\mathbf{u}_{\infty}\right)\right|^{2} \nabla_{x}\left(\mathbf{u}-\mathbf{u}_{\infty}\right)\right) \\
\mathbf{u}(0, x)=\mathbf{u}_{0}(x),\left.\mathbf{u}\right|_{I \times \partial \Omega}=\mathbf{u}_{B},
\end{gathered}
$$

with positive parameters $\varepsilon>0, \delta>0$, where we have denoted

$$
p_{\delta}(\varrho)=p(\varrho)+\delta \varrho^{\beta}, \quad \beta>\max \{\gamma, 9 / 2\}
$$

and where $\mathbf{u}_{\infty}$ is an extension of $\mathbf{u}_{B}$ defined in (2.13) 3 Next, following [3], we shall define the generalized solutions to the approximate problem (3.1 3.4) 4

Definition 3.1 Let $\mathbf{u}_{\infty} \in W^{1, \infty}\left(\Omega ; \mathbb{R}^{3}\right)$ be a Lipschitz extension of $\mathbf{u}_{B}$ staisfying (2.6), A couple $\left(\varrho_{\varepsilon}, \mathbf{u}_{\varepsilon}\right)$ and associated tensor field $\mathbb{Z}_{\varepsilon}$ is a generalized solution of the sequence of problems (3.1 3.4) $)_{\varepsilon>0}$ iff the following holds:

1. It belongs to the functional spaces:

$$
\begin{gathered}
\varrho_{\varepsilon} \in L^{\infty}\left(0, T ; L^{\beta}(\Omega)\right) \cap L^{2}\left(0, T ; W^{1,2}(\Omega)\right), 0 \leq \varrho_{\varepsilon} \text { a.e. in }(0, T) \times \Omega, \\
\mathbf{v}_{\varepsilon}:=\mathbf{u}_{\varepsilon}-\mathbf{u}_{\infty} \in L^{2}\left(0, T ; W_{0}^{1,2}\left(\Omega ; R^{3}\right)\right) \cap L^{4}\left(0, T ; W_{0}^{1,4}\left(\Omega ; R^{3}\right)\right), \\
\mathbb{Z}_{\varepsilon} \rightarrow 0 \text { in } L^{4 / 3}\left(Q_{T} ; \mathbb{R}^{3}\right) \text { as } \varepsilon \rightarrow 0 .
\end{gathered}
$$

2. Function $\varrho_{\varepsilon} \in C_{\text {weak }}\left([0, T], L^{\beta}(\Omega)\right)$ and the integral identity

$$
\begin{aligned}
& \int_{\Omega} \varrho_{\varepsilon}(\tau, x) \varphi(\tau, x) \mathrm{d} x-\int_{\Omega} \varrho_{0}(x) \varphi(0, x) \mathrm{d} x \\
& =\int_{0}^{\tau} \int_{\Omega}\left(\varrho_{\varepsilon} \partial_{t} \varphi+\varrho_{\varepsilon} \mathbf{u}_{\varepsilon} \cdot \nabla_{x} \varphi-\varepsilon \nabla_{x} \varrho_{\varepsilon} \cdot \nabla_{x} \varphi\right) \mathrm{d} x \mathrm{~d} t \\
& \quad-\int_{0}^{\tau} \int_{\Gamma_{\text {in }}} \varrho_{B} \mathbf{u}_{B} \cdot \mathbf{n} \varphi \mathrm{d} S_{x} \mathrm{~d} t-\int_{0}^{\tau} \int_{\Gamma_{\text {out }}} \varrho_{\varepsilon} \mathbf{u}_{B} \cdot \mathbf{n} \varphi \mathrm{d} S_{x} \mathrm{~d} t
\end{aligned}
$$

holds for any $\tau \in[0, T]$ and $\varphi \in C_{c}^{1}([0, T] \times \bar{\Omega})$.

\footnotetext{
${ }^{3}$ The exact choice of $\beta$ is irrelevant from the point of view of the final result provided it is sufficiently large.

${ }^{4}$ The only difference with respect to 3 in this definition is the fact, that the test function in the equation (3.7) does not vanish at the outflow boundary and the energy inequality is more precise containing also all boundary terms. This is essential for the construction of dissipative solutions.

${ }^{5}$ For bounded Lipschitz domains such extension always eists, cf. Remark 2.1
} 
3. Function $\varrho_{\varepsilon} \mathbf{u}_{\varepsilon} \in C_{\text {weak }}\left([0, T], L^{\frac{2 \beta}{\beta+1}}\left(\Omega ; \mathbb{R}^{3}\right)\right)$, and the integral identity

$$
\begin{array}{r}
\int_{\Omega} \varrho_{\varepsilon} \mathbf{v}_{\varepsilon}(\tau, \cdot) \cdot \boldsymbol{\varphi}(\tau, \cdot) \mathrm{d} x-\int_{\Omega} \varrho_{0} \mathbf{v}_{0}(\cdot) \boldsymbol{\varphi}(0, \cdot) \mathrm{d} x=-\int_{0}^{\tau} \int_{\Omega} \mathbb{Z}_{\varepsilon}: \nabla_{x} \varphi \mathrm{d} x \mathrm{~d} t \\
+\int_{0}^{\tau} \int_{\Omega}\left(\varrho_{\varepsilon} \mathbf{v}_{\varepsilon} \partial_{t} \varphi+\varrho_{\varepsilon} \mathbf{u}_{\varepsilon} \cdot \nabla_{x} \boldsymbol{\varphi} \cdot \mathbf{u}_{\varepsilon}-\varrho_{\varepsilon} \mathbf{u}_{\varepsilon} \cdot \nabla_{x}\left(\mathbf{u}_{\infty} \cdot \boldsymbol{\varphi}\right)+p_{\delta}\left(\varrho_{\varepsilon}\right) \operatorname{div}{ }_{x} \boldsymbol{\varphi}\right. \\
\left.\quad-\mathbb{S}\left(\nabla_{x} \mathbf{u}_{\varepsilon}\right): \nabla_{x} \boldsymbol{\varphi}+\varepsilon \nabla_{x} \varrho_{\varepsilon} \cdot \nabla_{x}\left(\mathbf{u}_{\infty} \cdot \boldsymbol{\varphi}\right)-\varepsilon \nabla_{x} \varrho_{\varepsilon} \cdot \nabla_{x} \mathbf{u}_{\varepsilon} \cdot \boldsymbol{\varphi}\right) \mathrm{d} x \mathrm{~d} t
\end{array}
$$

holds for any $\tau \in[0, T]$ and any $\varphi \in C_{c}^{1}\left([0, T] \times \Omega ; R^{3}\right)$.

4. Energy inequality

$$
\begin{aligned}
& \int_{\Omega}\left(\frac{1}{2} \varrho_{\varepsilon}\left|\mathbf{v}_{\varepsilon}\right|^{2}+H_{\delta}\left(\varrho_{\varepsilon}\right)\right) \mathrm{d} x+\int_{0}^{\tau} \int_{\Gamma_{\text {in }}} E_{\delta}\left(\varrho_{B} \mid \varrho_{\varepsilon}\right)\left|\mathbf{u}_{B} \cdot \mathbf{n}\right| \mathrm{d} S_{x} \mathrm{~d} t+\int_{0}^{\tau} \int_{\Gamma_{\text {out }}} H\left(\varrho_{\varepsilon}\right)\left|\mathbf{u}_{B} \cdot \mathbf{n}\right| \mathrm{d} S_{x} \mathrm{~d} t \\
& \quad+\int_{0}^{\tau} \int_{\Omega}\left(\mathbb{S}\left(\nabla_{x} \mathbf{u}_{\varepsilon}\right): \nabla_{x} \mathbf{v}_{\varepsilon}+\varepsilon H_{\delta}^{\prime \prime}\left(\varrho_{\varepsilon}\right)\left|\nabla_{x} \varrho_{\varepsilon}\right|^{2}+\varepsilon\left|\nabla_{x} \mathbf{v}_{\varepsilon}\right|^{4}\right) \mathrm{d} x \mathrm{~d} t \\
& \leq \int_{\Omega}\left(\frac{1}{2} \varrho_{0}\left|\mathbf{u}_{0}-\mathbf{u}_{\infty}\right|^{2}+H_{\delta}\left(\varrho_{0}\right)\right) \mathrm{d} x-\int_{0}^{\tau} \int_{\Gamma_{\text {in }}} H_{\delta}\left(\varrho_{B}\right) \mathbf{u}_{B} \cdot \mathbf{n} \mathrm{d} S_{x} \mathrm{~d} t \\
& \quad-\int_{0}^{\tau} \int_{\Omega}\left(p_{\delta}\left(\varrho_{\varepsilon}\right) \operatorname{div} \mathbf{u}_{\infty}+\varrho_{\varepsilon} \mathbf{u}_{\varepsilon} \cdot \nabla_{x} \mathbf{u}_{\infty} \cdot \mathbf{v}_{\varepsilon}-\varepsilon \nabla_{x} \varrho \cdot \nabla_{x} \mathbf{v}_{\varepsilon} \cdot \mathbf{u}_{\infty}\right) \mathrm{d} x \mathrm{~d} t
\end{aligned}
$$

holds for a.e. $\tau \in(0, T)$. In the above,

$$
H_{\delta}(\varrho)=H(\varrho)+\delta H^{(\beta)}(\varrho), H^{(\beta)}(\varrho)=\varrho \int_{1}^{\varrho} z^{\beta-2} \mathrm{~d} z=\frac{1}{\beta-1} \varrho^{\beta}
$$

and

$$
E_{\delta}(\varrho \mid r)=E(\varrho \mid r)+\delta E^{(\beta)}(\varrho \mid r), E^{(\beta)}(\varrho \mid r)=H^{(\beta)}(\varrho)-\left[H^{(\beta)}\right]^{\prime}(r)(\varrho-r)-H^{(\beta)}(r) .
$$

The starting point in the construction of the dissipative solutions for system (1.1 1.5) is the existence theorem for the generalized approximating problem (3.13.4). It is announced in the next lemma. 6

Lemma 3.1. Let $\Omega$ be a domain of class $C^{2}$. Let $\left(\varrho_{B}, \mathbf{u}_{B}\right)$ verify assumptions (2.1) and let initial and boundary data verify

$$
\begin{gathered}
\mathbf{u}_{0} \in L^{2}(\Omega), \quad \varrho_{0} \in W^{1,2}(\Omega), 0<\underline{\varrho} \leq \varrho_{0} \leq \bar{\varrho}<\infty, \\
0<\underline{\varrho}_{B} \leq \varrho_{B} \leq \bar{\varrho}_{B}<\infty .
\end{gathered}
$$

Then for any continuous extension $\mathbf{u}_{\infty}$ of $\mathbf{u}_{B}$ in class (2.13) there exists a generalized solution $\left(\varrho_{\epsilon}, \mathbf{u}_{\epsilon}\right)$ and $\mathbb{Z}_{\varepsilon}$ to the sequence of approximate problems (3.1 - 3.4) $)_{\varepsilon \in(0,1)}$ - which belongs to the functional spaces (3.6), satisfies the weak formulations (3.7 3.8) and verifies the energy inequality (3.9) - with the following extra properties:

${ }^{6}$ The only difference with respect to [3. Lemma 4.1] in Lemma 3.1 is the fact, that the test function in equation (3.7) do not vanish at the outflow boundary and the energy inequality is more precise containing also all boundary terms. This is essential for the construction of dissipative solutions. 
(i) In addition to (3.6) it belongs to functional spaces:

$$
\varrho_{\varepsilon} \in L^{\frac{5}{3} \beta}\left(Q_{T}\right), \sqrt{\varrho_{\varepsilon}}, \varrho_{\epsilon}^{\frac{\beta}{2}} \in L^{2}\left(I, W^{1,2}(\Omega)\right), \partial_{t} \varrho_{\epsilon} \in L^{4 / 3}\left(Q_{T}\right), \nabla^{2} \varrho_{\epsilon} \in L^{4 / 3}\left(Q_{T}\right) .
$$

(ii) In addition to the weak formulation (3.7), the couple $\left(\varrho_{\varepsilon}, \mathbf{u}_{\varepsilon}\right)$ satisfies the equation (3.7) in the strong sense, meaning, it verifies equation (3.1) with $\left(\varrho_{\varepsilon}, \mathbf{u}_{\varepsilon}\right)$ a.e. in $Q_{T}$, boundary identity (3.2) with $\left(\varrho_{\varepsilon}, \mathbf{u}_{\varepsilon}\right)$ a.e. in $(0, T) \times \partial \Omega$ and initial conditions in the sense $\lim _{t \rightarrow 0+}\left\|\varrho_{\varepsilon}(t)-\varrho_{0}\right\|_{L^{4 / 3}(\Omega)}=0$.

(iii) The couple $\left(\varrho_{\varepsilon}, \mathbf{u}_{\varepsilon}\right)$ satisfies identity

$$
\partial_{t} b\left(\varrho_{\varepsilon}\right)+\varepsilon b^{\prime \prime}\left(\varrho_{\varepsilon}\right)\left|\nabla_{x} \varrho_{\varepsilon}\right|^{2}-\varepsilon \operatorname{div}_{x}\left(b^{\prime}\left(\varrho_{\varepsilon}\right) \nabla_{x} \varrho_{\varepsilon}\right)+\operatorname{div}_{x}\left(b\left(\varrho_{\varepsilon}\right) \mathbf{u}_{\varepsilon}\right)+\left[b^{\prime}\left(\varrho_{\varepsilon}\right) \varrho_{\varepsilon}-b\left(\varrho_{\varepsilon}\right)\right] \operatorname{div}_{x} \mathbf{u}_{\varepsilon}=0
$$

a.e. in $(0, T) \times \Omega$ with any $b \in C^{2}[0, \infty)$, where the space-time derivatives have to be understood in the sense a.e.

Remark 3.2. Identity (3.15) holds in the weak sense

$$
\begin{gathered}
\int_{\Omega} b\left(\varrho_{\varepsilon}(\tau)\right) \varphi(\tau) \mathrm{d} x-\int_{\Omega} b\left(\varrho_{0}\right) \varphi(0) \mathrm{d} x=\int_{0}^{\tau} \int_{\partial \Omega}\left(\varepsilon b^{\prime}\left(\varrho_{\varepsilon}\right) \nabla_{x} \varrho_{\varepsilon}-b\left(\varrho_{\varepsilon}\right) \mathbf{u}_{\varepsilon}\right) \cdot \mathbf{n} \mathrm{d} S_{x} \mathrm{~d} t \\
+\int_{0}^{\tau} \int_{\Omega}\left[b\left(\varrho_{\varepsilon}\right) \partial_{t} \varphi+\left(b\left(\varrho_{\varepsilon}\right) \mathbf{u}_{\varepsilon}-\varepsilon b^{\prime}\left(\varrho_{\varepsilon}\right) \nabla_{x} \varrho_{\varepsilon}\right) \cdot \nabla_{x} \varphi-\varphi\left(\varepsilon b^{\prime \prime}\left(\varrho_{\varepsilon}\right)\left|\nabla_{x} \varrho_{\varepsilon}\right|^{2}+\left(\varrho_{\varepsilon} b^{\prime}\left(\varrho_{\varepsilon}\right)-b\left(\varrho_{\varepsilon}\right)\right) \operatorname{div} \mathbf{u}_{\varepsilon}\right)\right] \mathrm{d} x \mathrm{~d} t
\end{gathered}
$$

with any $\tau \in[0, T]$ and $\varphi \in C_{c}^{1}([0, T] \times \bar{\Omega})$ with any $b$ whose growth (and that one of its derivatives) in combination with (3.14) guarantees $b\left(\varrho_{\varepsilon}\right) \in C_{\text {weak }}\left([0, T] ; L^{1}(\Omega)\right)$, existence of traces and integrability of all terms appearing at the r.h.s.

\section{Construction of the generalized solutions to the approxi- mate problem}

We recall here the main building blocks of the construction of generalized solutions to the approximate problem (3.1 3.4). We do not intend to describe the whole process in all details, since it is available in [3, Section 4], but only its main parts.

\subsection{Galerkin type approximation and energy inequality}

1. The first building block in the construction of generalized solutions to the approximate problem (3.1] 3.4) is the following theorem dealing with the parabolic problem (3.1] 3.2). It reads, cf. [3, Lemma 4.3]:

Lemma 4.1. Suppose that $\Omega$ is a bounded domain of class $C^{2}$ and assume further that $\varrho_{0} \in W^{1,2}(\Omega)$, $\left.\mathbf{u}\right|_{(0, T) \times \partial \Omega}=\mathbf{u}_{B}, v, g \in C^{1}(\partial \Omega)$. Then we have: 
1. The parabolic problem (3.1 3.2) admits for any $\mathbf{u} \in L^{\infty}\left(0, T ; W^{1, \infty}(\Omega)\right)$, a unique solution $\varrho=$ $S(\mathbf{u})$ in the class

$$
\varrho \in L^{2}\left(0, T ; W^{2,2}(\Omega)\right) \cap W^{1,2}\left(0, T ; L^{2}(\Omega)\right) .
$$

2. Suppose that

$$
\underline{\varrho} \leq \varrho_{0}(x) \leq \bar{\varrho} \text { for } a \text {. a. } x \in \Omega, \quad \underline{\varrho} \leq \varrho_{B}(x) \leq \bar{\varrho} \text { for all } x \in \Gamma_{\text {in }} .
$$

Then

$$
\underline{\varrho} \exp \left(-\int_{0}^{\tau}\|\operatorname{div} \mathbf{u}(s)\|_{L^{\infty}(\Omega)} \mathrm{d} s\right) \leq \varrho(\tau, x) \leq \bar{\varrho} \exp \left(\int_{0}^{\tau}\|\operatorname{div} \mathbf{u}(s)\|_{L^{\infty}(\Omega)} \mathrm{d} s\right)
$$

in particular,

$$
\underline{\varrho} e^{-K \tau} \leq \varrho(t, x) \leq \bar{\varrho} e^{K \tau}
$$

for all $\tau \in[0, T]$ provided $\mathbf{u}$ verifies condition

$$
\|\mathbf{u}\|_{L^{\infty}\left(Q_{T}\right)}+\|\operatorname{div} \mathbf{u}\|_{L^{\infty}\left(Q_{T}\right)} \leq K
$$

2. The second building block in the construction of generalized solutions to the approximate problem (3.13.4) is the Galekin approximation of the momentum equation (3.3 3.4). In order to write it down, we introduce

$$
\left.X=\operatorname{span}\left\{\Phi_{i}\right\}_{i=1}^{N} \text { where } \mathcal{B}:=\left\{\Phi_{i} \in C_{c}^{\infty}(\Omega) \mid i \in \mathbb{N}^{*}\right\} \text { is an orthonormal basis in } L^{2}\left(\Omega ; \mathbb{R}^{3}\right)\right\},
$$

a finite dimensional real Hilbert space with scalar product $(\cdot, \cdot)_{X}$ induced by the scalar product in $L^{2}\left(\Omega ; R^{3}\right)$ and $\|\cdot\|_{X}$ the norm induced by this scalar product. We denote by $P_{N}$ the orthogonal projection of $L^{2}\left(\Omega ; R^{3}\right)$ to $X$.

The Galerkin approximation of system (3.13.4) reads: Given $\varrho_{B}, \mathbf{u}_{\infty}$ (whence also $\mathbf{u}_{B}$ ), and $\mathbf{u}_{0}=$ $\mathbf{v}_{0}-\mathbf{u}_{\infty}, \varrho_{0}$, find the couple $(\varrho, \mathbf{u}), \mathbf{u}=\mathbf{v}+\mathbf{u}_{\infty}$

$$
\mathbf{v} \in C(\bar{I} ; X), \partial_{t} \mathbf{v} \in L^{2}(I ; X), 0 \leq \varrho \in L^{2}\left(I ; W^{2,2}(\Omega)\right) \cap W^{1,2}\left(I ; L^{2}(\Omega)\right)
$$

such that

$$
\begin{gathered}
\int_{\Omega} \varrho \mathbf{v}(t) \cdot \Phi \mathrm{d} x-\int_{\Omega} \varrho_{0} \mathbf{v}_{0} \Phi \mathrm{d} x=\int_{0}^{t} \int_{\Omega}\left(\operatorname{div} \mathbb{S}\left(\nabla_{x} \mathbf{u}\right)+\varepsilon \operatorname{div}\left(\left|\nabla_{x}\left(\mathbf{u}-\mathbf{u}_{\infty}\right)\right|^{2} \nabla_{x}\left(\mathbf{u}-\mathbf{u}_{\infty}\right)\right)\right. \\
\left.-\nabla_{x} p_{\delta}(\varrho)-\operatorname{div}(\varrho \mathbf{u} \otimes \mathbf{u})-\varepsilon \nabla_{x} \varrho \cdot \nabla_{x} \mathbf{u}-\partial_{t} \varrho \mathbf{u}_{\infty}\right) \cdot \Phi \mathrm{d} x \mathrm{~d} t
\end{gathered}
$$

holds for all $t \in[0, T]$ with any $\Phi \in X$, where $\mathbf{u}=\mathbf{v}+\mathbf{u}_{\infty}$ and

$$
\text { @ solves parabolic problem (3.1 3.2). }
$$

The next lemma ensures existence of solutions to problem (4.54.7). 
Lemma 4.2. Let $\Omega,\left(\varrho_{0}, \mathbf{u}_{0}\right),\left(\varrho_{B}, \mathbf{u}_{B}\right)$ verify assumptions of Lemma 3.1 .

1. Then for any continuous extension $\mathbf{u}_{\infty}$ of $\mathbf{u}_{B}$ in class (2.13) the Galerkin type problem (4.6 4.7) admits a unique solution $(\varrho, \mathbf{v})$ in class (4.5). Moreover, there are constants $0<\underline{c}=\underline{c}(N)<\bar{c}=$ $\bar{c}(N)$ such that

$$
\forall t \in \bar{I}, \underline{c} \leq \varrho(t, \cdot) \leq \bar{c} \text { a.e. in } \Omega .
$$

2. The solution satisfies energy inequality:

$$
\begin{aligned}
& \int_{\Omega}\left(\frac{1}{2} \varrho|\mathbf{v}|^{2}+H_{\delta}(\varrho)\right)(\tau) \mathrm{d} x+\int_{0}^{\tau} \int_{\Gamma_{\mathrm{in}}} E_{\delta}\left(\varrho_{B} \mid \varrho\right)\left|\mathbf{u}_{B} \cdot \mathbf{n}\right| \mathrm{d} S_{x} \mathrm{~d} t+\int_{0}^{\tau} \int_{\Gamma_{\text {out }}} H_{\delta}(\varrho)\left|\mathbf{u} \mathbf{u}_{B} \cdot \mathbf{n}\right| \mathrm{d} S_{x} \mathrm{~d} t \\
& \quad+\varepsilon \int_{0}^{\tau} \int_{\Omega}\left|\nabla_{x} \mathbf{v}\right|^{4} \mathrm{~d} x \mathrm{~d} t+\varepsilon \int_{0}^{\tau} \int_{\Omega} H_{\delta}^{\prime \prime}(\varrho)\left|\nabla_{x} \varrho\right|^{2} \mathrm{~d} x \mathrm{~d} t+\int_{0}^{\tau} \int_{\Omega} \mathbb{S}\left(\nabla_{x} \mathbf{u}\right): \nabla_{x} \mathbf{v} \mathrm{d} x \mathrm{~d} t \\
& \leq \int_{\Omega}\left(\frac{1}{2} \varrho_{0}\left|\mathbf{v}_{0}\right|^{2}+H_{\delta}\left(\varrho_{0}\right)\right) \mathrm{d} x-\int_{0}^{\tau} \int_{\Gamma_{\text {in }}} H_{\delta}\left(\varrho_{B}\right) \mathbf{u}_{B} \cdot \mathbf{n} \mathrm{d} S_{x} \mathrm{~d} t \\
& \quad+\int_{0}^{\tau} \int_{\Omega}\left(-p_{\delta}(\varrho) \operatorname{div} \mathbf{u}_{\infty}-\varrho \mathbf{u} \cdot \nabla_{x} \mathbf{u}_{\infty} \cdot \mathbf{v}+\varepsilon \nabla_{x} \varrho \cdot \nabla_{x} \mathbf{v} \cdot \mathbf{u}_{\infty}\right) \mathrm{d} x \mathrm{~d} t
\end{aligned}
$$

where $H_{\delta}$ and $E_{\delta}$ are defined in (3.10) and (3.11).

\section{Proof of Lemma 4.2}

We refer to [3, Section 4.3] for the proof of the existence part (Item 1.). Energy inequality (4.9) - Item 2. - is derived in formula (4.40) of the same paper. We repeat its proof here for the sake of completeness, since the energy inequality plays crucial role in the genesis of the proof of the exact energy inequality (3.9) and consequently in the proof of the existence of dissipative solutions.

We have at our disposal the couple $\left(\varrho=\varrho_{N}=S\left(\mathbf{u}_{N}\right), \mathbf{u}=\mathbf{u}_{N}\right)$ a solution of problem (4.6 4.7) in the class (4.5) and (4.8). We are going to derive for this couple the energy inequality (4.9).

First, we multiply equation (4.7) by $H_{\delta}^{\prime}(\varrho)$ and integrate over $\Omega$ to deduce

$$
\begin{aligned}
& \partial_{t} \int_{\Omega} H_{\delta}(\varrho) \mathrm{d} x+\varepsilon \int_{\Omega} H_{\delta}^{\prime \prime}(\varrho)\left|\nabla_{x} \varrho\right|^{2} \mathrm{~d} x \\
& \quad+\int_{\Gamma_{\mathrm{in}}}\left(H_{\delta}\left(\varrho_{B}\right)-H_{\delta}^{\prime}(\varrho)\left(\varrho_{B}-\varrho\right)-H_{\delta}(\varrho)\right)|v| \mathrm{d} S_{x}+\int_{\Gamma_{\text {out }}} H_{\delta}(\varrho) \mathbf{u}_{B} \cdot \mathbf{n} \mathrm{d} S_{x} \\
& =-\int_{\Omega} p_{\delta}(\varrho) \operatorname{div} \mathbf{u} \mathrm{d} x-\int_{\Gamma_{\mathrm{in}}} H_{\delta}\left(\varrho_{B}\right) \mathbf{u}_{B} \cdot \mathbf{n} \mathrm{d} S_{x},
\end{aligned}
$$

where we have used several times integration by parts, take into account boundary conditions (4.7), and where $\mathbf{v}=\mathbf{u}-\mathbf{u}_{\infty}$.

Further, we deduce from (4.6)

$$
\int_{0}^{\tau} \int_{\Omega}\left(\partial_{t}(\varrho \mathbf{v}) \cdot \mathbf{v}-\varrho \mathbf{u} \otimes \mathbf{u}: \nabla_{x} \mathbf{v}\right) \mathrm{d} x \mathrm{~d} t+\int_{0}^{\tau} \int_{\Omega}\left(\mathbb{S}\left(\nabla_{x} \mathbf{u}\right): \nabla_{x} \mathbf{v}+\varepsilon\left|\nabla_{x} \mathbf{v}\right|^{4}\right) \mathrm{d} x \mathrm{~d} t
$$




$$
-\int_{0}^{\tau} \int_{\Omega} p_{\delta}(\varrho) \operatorname{div} \mathbf{v} \mathrm{d} x \mathrm{~d} t+\int_{0}^{\tau} \int_{\Omega} \varepsilon \nabla_{x} \varrho \cdot \nabla_{x} \mathbf{u} \cdot \mathbf{v} \mathrm{d} x \mathrm{~d} t+\int_{0}^{\tau} \int_{\Omega} \partial_{t} \varrho \mathbf{u}_{\infty} \cdot \mathbf{v} \mathrm{d} x \mathrm{~d} t=0,
$$

where by virtue of (4.7) (after several integrations by parts and recalling that $\mathbf{u}=\mathbf{u}_{\infty}+\mathbf{v}$ ),

$$
\begin{aligned}
\int_{\Omega}\left(\partial_{t}(\varrho \mathbf{v})\right. & \left.\cdot \mathbf{v}-\varrho \mathbf{u} \otimes \mathbf{u}: \nabla_{x} \mathbf{v}\right) \mathrm{d} x \\
= & \int_{\Omega}\left(\partial_{t} \varrho \mathbf{v}^{2}+\frac{1}{2} \varrho \partial_{t}\left(\mathbf{v}^{2}\right)+\frac{1}{2} \operatorname{div}(\varrho \mathbf{u}) \mathbf{v}^{2}-\varrho \mathbf{u} \cdot \nabla_{x} \mathbf{v} \cdot \mathbf{u}_{\infty}\right) \mathrm{d} x \\
& =\int_{\Omega}\left(\frac{1}{2} \partial_{t}\left(\varrho \mathbf{v}^{2}\right)-\varepsilon \nabla_{x} \varrho \cdot \nabla_{x} \mathbf{v} \cdot \mathbf{v}-\varrho \mathbf{u} \cdot \nabla_{x} \mathbf{v} \cdot \mathbf{u}_{\infty}\right) \mathrm{d} x
\end{aligned}
$$

and

$$
\int_{\Omega} \partial_{t} \varrho \mathbf{u}_{\infty} \cdot \mathbf{v} \mathrm{d} x=\int_{\Omega}\left(\varrho \mathbf{u} \cdot \nabla_{x} \mathbf{v} \cdot \mathbf{u}_{\infty}+\varrho \mathbf{u} \cdot \nabla_{x} \mathbf{u}_{\infty} \cdot \mathbf{v}-\varepsilon \nabla_{x} \varrho \cdot \nabla_{x} \mathbf{u}_{\infty} \cdot \mathbf{v}-\varepsilon \nabla_{x} \varrho \cdot \nabla_{x} \mathbf{v} \cdot \mathbf{u}_{\infty}\right) \mathrm{d} x .
$$

Therefore,

$$
\begin{gathered}
\left.\int_{0}^{\tau} \int_{\Omega} \partial_{t}(\varrho \mathbf{v}) \cdot \mathbf{v}-\varrho \mathbf{u} \otimes \mathbf{u}: \nabla_{x} \mathbf{v}+\varepsilon \nabla_{x} \varrho \cdot \nabla_{x} \mathbf{u} \cdot \mathbf{v}+\partial_{t} \varrho \mathbf{u}_{\infty} \cdot \mathbf{v}\right) \mathrm{d} x \\
=\int_{0}^{\tau} \int_{\Omega}\left(\varrho \mathbf{u} \cdot \nabla_{x} \mathbf{u}_{\infty} \cdot \mathbf{v}-\varepsilon \nabla_{x} \varrho \cdot \nabla_{x} \mathbf{v} \cdot \mathbf{u}_{\infty}\right) \mathrm{d} x \mathrm{~d} t
\end{gathered}
$$

This together with (4.10) yields inequality (4.9). Lemma 4.2 is thus proved.

\subsection{Uniform bounds with respect to $N$ and limit $N \rightarrow \infty$}

In order to get uniform bounds for the sequence $\left(\varrho_{N}, \mathbf{u}_{N}\right)$ we still need the conservation of mass

$$
\int_{\Omega} \varrho(\tau) \mathrm{d} x+\int_{0}^{\tau} \int_{\Gamma_{\text {out }}} \varrho \mathbf{u}_{B} \cdot \mathbf{n} \mathrm{d} S_{x} \mathrm{~d} t=\int_{\Omega} \varrho_{0} \mathrm{~d} x+\int_{0}^{\tau} \int_{\Gamma_{\text {in }}} \varrho\left|\mathbf{u}_{B} \cdot \mathbf{n}\right| \mathrm{d} S_{x} \mathrm{~d} t
$$

(which we obtain from (4.7)). We also need to to test equation (3.1) ${\left(\varrho_{N}, \mathbf{u}_{N}\right)}$ by $\varrho_{N}$ in order to get, after several integrations by parts,

$$
\begin{aligned}
& \frac{1}{2} \int_{\Omega} \varrho^{2}(\tau) \mathrm{d} x+\frac{1}{2} \int_{0}^{\tau} \int_{\partial \Omega} \varrho^{2}\left|\mathbf{u}_{B} \cdot \mathbf{n}\right| \mathrm{d} S_{x} \mathrm{~d} t+\varepsilon \int_{0}^{\tau} \int_{\Omega}\left|\nabla_{x} \varrho\right|^{2} \mathrm{~d} x \mathrm{~d} t \\
& \quad=\frac{1}{2} \int_{\Omega} \varrho_{0}^{2} \mathrm{~d} x+\int_{0}^{\tau} \int_{\Gamma_{\text {in }}} \varrho \varrho_{B}\left|\mathbf{u}_{B} \cdot \mathbf{n}\right| \mathrm{d} S_{x} \mathrm{~d} t-\frac{1}{2} \int_{0}^{\tau} \int_{\Omega} \varrho^{2} \operatorname{div} \mathbf{u} \mathrm{d} x \mathrm{~d} t
\end{aligned}
$$

Recalling structural assumptions (2.2), (2.18) for $p$, definitions (2.3), (3.5), (3.10), we deduce from the energy inequality (4.9) and inequalities (4.114.12) the following uniform bounds with respect to $N$ for the sequence $\left(\varrho_{N}=S\left(\mathbf{u}_{N}\right), \mathbf{u}_{N}=\mathbf{u}_{\infty}+\mathbf{v}_{N}\right)$ of Galerkin solutions to the problem (4.6 4.7):

$$
\left\|\varrho_{N}\left|\mathbf{u}_{N}\right|^{2}\right\|_{L^{\infty}\left(I, L^{1}(\Omega)\right)} \leq L(\text { data })
$$




$$
\begin{aligned}
& \left\|\mathbf{u}_{N}\right\|_{L^{2}\left(I, W^{1,2}(\Omega)\right)} \leq L(\text { data }), \\
& \left\|\varrho_{N}\right\|_{L^{\infty}\left(I, L^{\gamma}(\Omega)\right)} \leq L(\text { data }), \\
& \delta^{1 / \beta}\left\|\varrho_{N}\right\|_{L^{\infty}\left(I, L^{\beta}(\Omega)\right)} \leq L(\text { data }), \\
& \varepsilon\left\|\nabla \varrho_{N}\right\|_{L^{2}\left(Q_{T}\right)}^{2}+\varepsilon\left\|\nabla\left(\varrho_{N}^{\beta / 2}\right)\right\|_{L^{2}\left(Q_{T}\right)}^{2} \leq L(\text { data }, \delta), \\
& \varepsilon^{1 / \beta} \| \varrho_{L^{\beta}((0, T) \times \partial \Omega)} \leq L(\text { data }, \delta), \\
& \varepsilon\left\|\mathbf{u}_{N}-\mathbf{u}_{\infty}\right\|_{L^{4}\left(0, T ; W^{1,4}(\Omega)\right)}^{4} \leq L(\text { data }, \delta) .
\end{aligned}
$$

In the above and hereafter

$$
\text { "data" stands for } \int_{\Omega}\left(\frac{1}{2} \varrho_{0}\left|\mathbf{v}_{0}\right|^{2}+H\left(\varrho_{0}\right)\right) \mathrm{d} x,\left\|\mathbf{u}_{\infty}\right\|_{W^{1, \infty}(\Omega)}, \underline{\varrho}, \underline{\varrho}, \underline{\varrho}_{B}, \bar{\varrho}_{B}, \underline{H}=\inf _{\varrho>0} H(\varrho) .
$$

Due to the above estimates, expression $\operatorname{div}\left(\varrho_{N} \mathbf{u}_{N}\right)$ is bounded in $L^{4 / 3}\left(Q_{T}\right)$. We can thus return to equation (3.1 3.2) -with $\left(\varrho_{N}, \mathbf{u}_{N}\right)$ - and consider it as parabolic problem with operator $\partial_{t} \varrho-\varepsilon \Delta \varrho$ in $(0, T) \times \Omega$ with right hand side $-\operatorname{div}\left(\varrho_{N} \mathbf{u}_{N}\right)$, and boundary operator $-\varepsilon \mathbf{n} \cdot \nabla_{x} \varrho+v \varrho$ in $(0, T) \times \partial \Omega$ with right hand side $\varrho_{B} v$. The maximal parabolic regularity theory, see e.g. [7, Theorem 2.1], yields that

$$
\left\|\partial_{t} \varrho_{N}\right\|_{L^{4 / 3}\left(Q_{T}\right)}+\left\|\varrho_{N}\right\|_{L^{4 / 3}\left(0, T ; W^{2,4 / 3}(\Omega)\right)} \leq L(\text { data, } \delta, \varepsilon) .
$$

Estimates (4.13 4.20) yield, in particular, existence of a subsequence $\left(\varrho_{N}, \mathbf{u}_{N}\right)$ not relabeled such that

$$
\begin{aligned}
& \varrho_{N} \rightarrow \varrho \text { in } L^{4 / 3}\left(0, T ; W^{2,4 / 3}(\Omega)\right) \text { and in } L^{2}\left(0, T ; W^{1,2}(\Omega)\right), \quad \partial_{t} \varrho_{N} \rightarrow \partial_{t} \varrho \text { in } L^{4 / 3}\left(Q_{T}\right) \\
& \varrho_{N} \rightarrow \varrho \text { in } L^{\beta}((0, T) \times \partial \Omega), \\
& \mathbf{u}_{N} \rightarrow \mathbf{u} \text { (weakly) in } L^{4}\left(0, T ; W^{1,4}(\Omega)\right), \\
& \varepsilon\left|\nabla_{x}\left(\mathbf{u}_{N}-\mathbf{u}_{\infty}\right)\right|^{2} \nabla_{x}\left(\mathbf{u}_{N}-\mathbf{u}_{\infty}\right) \rightarrow \mathbb{Z} \equiv \mathbb{Z}_{\varepsilon} \text { weakly in } L^{4 / 3}\left(Q_{T} ; R^{9}\right),
\end{aligned}
$$

where

$$
\left\|\mathbb{Z}_{\varepsilon}\right\|_{L^{4 / 3}\left(Q_{T}\right)} \rightarrow 0 \text { as } \varepsilon \rightarrow 0 .
$$

This information is enough to show that $(\varrho, \mathbf{u}, \mathbb{Z})$ to class (3.6), (3.14) and allows to pass to the limit $N \rightarrow \infty$ in the system (4.6 4.7) and in the inequality (4.9) by using only the standard compactness arguments (which are Sobolev embeddings, Arzela-Ascoli theorem and Lions-Aubin Lemma). In particular, relation (4.21) guarantees that equation (3.7) is satisfied in the strong sense (3.1). Equation (3.15) is obtained by multiplying (3.1) by $b^{\prime}(\varrho)$. To pass to the limit from inequality (4.9) $\left(_{(}, \mathbf{u}_{N}\right)$ to inequality (3.9) one uses, at the left-hand side the lower weak semi-continuity of convex functionals. The details of this limit passage are available in [3, Section 4.3.4]. We have thus established Lemma 3.1.

\section{Relative energy for the approximate system}

In this Section, we shall derive a convenient form of the relative energy inequality for any generalized solution of the approximate system (3.1 3.4). This result is subject of the following lemma. 
Lemma 5.1. Let all assumptions of Lemma 3.1 be satisfied. Let $\left(\varrho=\varrho_{\varepsilon}, \mathbf{u}=\mathbf{u}_{\varepsilon}\right)$ and an associted tensor field $\mathbb{Z}=\mathbb{Z}_{\varepsilon}$ be a generalized solution to problem (3.1 3.4) (3>0 constructed in Lemma 3.1. Then $\varrho, \mathbf{u}, \mathbb{Z}$ satisfy the so called relative energy inequality

$$
\begin{aligned}
& \int_{\Omega}\left(\frac{1}{2} \varrho|\mathbf{v}-\mathbf{V}|^{2}+E_{\delta}(\varrho \mid r)\right)(\tau, x) \mathrm{d} x \\
& \quad+\int_{0}^{\tau} \int_{\Gamma_{\text {in }}} E_{\delta}\left(\varrho_{B} \mid \varrho\right)\left|\mathbf{u}_{B} \cdot \mathbf{n}\right| \mathrm{d} S_{x} \mathrm{~d} t+\int_{0}^{\tau} \int_{\Gamma_{\text {out }}} E_{\delta}(\varrho \mid r) \mathbf{u}_{B} \cdot \mathbf{n} \mathrm{d} S_{x} \mathrm{~d} t \\
& \quad+\int_{0}^{\tau} \int_{\Omega}\left(\mathbb{S}\left(\nabla_{x} \mathbf{u}\right): \nabla_{x}(\mathbf{v}-\mathbf{V})+\varepsilon H_{\delta}^{\prime \prime}(\varrho)\left|\nabla_{x} \varrho\right|^{2}+\varepsilon\left|\nabla_{x} \mathbf{V}\right|^{4}\right) \mathrm{d} x \mathrm{~d} t \\
& \leq \int_{\Omega}\left(\frac{1}{2} \varrho_{0}\left|\mathbf{v}_{0}-\mathbf{V}(0, \cdot)\right|^{2}+E_{\delta}\left(\varrho_{0} \mid r(0, \cdot)\right) \mathrm{d} x\right. \\
& \quad+\int_{0}^{\tau} \int_{\Gamma_{\text {in }}}\left(H_{\delta}(r)-r H_{\delta}^{\prime}(r)-H_{\delta}\left(\varrho_{B}\right)+\varrho_{B} H_{\delta}^{\prime}(r)\right) \mathbf{u}_{B} \cdot \mathbf{n} \mathrm{d} S_{x} \mathrm{~d} t \\
& \quad+\int_{0}^{\tau} \int_{\Omega}\left(\varrho(\mathbf{V}-\mathbf{v}) \cdot \partial_{t} \mathbf{V}+\varrho \mathbf{u} \cdot \nabla_{x} \mathbf{U} \cdot(\mathbf{V}-\mathbf{v})\right. \\
& \quad+\left(p_{\delta}(r)-p_{\delta}^{\prime}(r)(r-\varrho)-p_{\delta}(\varrho)\right) \mathrm{div} \mathbf{U} \\
& \left.\quad+\frac{r-\varrho}{r} p_{\delta}^{\prime}(r)(\mathbf{v}-\mathbf{V}) \cdot \nabla_{x} r-p_{\delta}^{\prime}(r) \mathbf{v} \cdot \nabla_{x} r-p_{\delta}(r) \operatorname{div} \mathbf{V}\right) \mathrm{d} x \mathrm{~d} t \\
& \quad+\int_{0}^{\tau} \int_{\Omega}\left(\mathbb{Z}: \nabla_{x} \mathbf{V}+\varepsilon \nabla_{x} \varrho \cdot \nabla_{x}(\mathbf{u}-\mathbf{V}) \cdot \mathbf{V}\right) \mathrm{d} x \mathrm{~d} t,
\end{aligned}
$$

with a.e. $\tau \in \bar{I}$ and with any couple $(r, \mathbf{U})$ belonging to class (2.112.12). In the above $\mathbf{V}=\mathbf{U}-\mathbf{u}_{\infty}$, $\mathbf{v}=\mathbf{u}-\mathbf{u}_{\infty}$.

\section{Proof of Lemma 5.1}

We take any couple $(r, \mathbf{U})$ (and $\mathbf{V}$ related to $\mathbf{U}$ ) belonging to class (2.112.12).

1. Using in the regularized continuity equation (3.7) the test function $\varphi=\frac{1}{2}|\mathbf{V}|^{2}$, we get:

$$
\begin{aligned}
\int_{\Omega} \frac{1}{2} \varrho(\tau, x)|\mathbf{V}|^{2}(\tau, x) \mathrm{d} x-\int_{\Omega} \frac{1}{2} \varrho_{0}(x)|\mathbf{V}|^{2}(0, x) \mathrm{d} x \\
\quad=\int_{0}^{\tau} \int_{\Omega}\left(\varrho \mathbf{V} \cdot \partial_{t} \mathbf{V}+\varrho \mathbf{u} \cdot \nabla_{x} \mathbf{V} \cdot \mathbf{V}-\varepsilon \nabla_{x} \varrho \cdot \nabla_{x} \mathbf{V} \cdot \mathbf{V}\right) \mathrm{d} x \mathrm{~d} t
\end{aligned}
$$

for all $\tau \in \bar{I}$.

2. Using in the momentum equation (3.8) the test function $\varphi=-\mathbf{V}$, we obtain:

$$
-\int_{\Omega} \varrho \mathbf{v}(\tau, \cdot) \cdot \mathbf{V}(\tau, \cdot) \mathrm{d} x+\int_{\Omega} \varrho_{0} \mathbf{v}_{0}(\cdot) \mathbf{V}(0, \cdot) \mathrm{d} x=\int_{0}^{\tau} \int_{\Omega} \mathbb{Z}_{\varepsilon}: \nabla_{x} \varphi \mathrm{d} x \mathrm{~d} t
$$




$$
\begin{aligned}
& +\int_{0}^{\tau} \int_{\Omega}\left(-\varrho \mathbf{v} \partial_{t} \mathbf{V}-\varrho \mathbf{u} \cdot \nabla_{x} \mathbf{V} \cdot \mathbf{u}+\varrho \mathbf{u} \cdot \nabla_{x}\left(\mathbf{u}_{\infty} \cdot \mathbf{V}\right)-p_{\delta}(\varrho) \operatorname{div}_{x} \mathbf{V}\right. \\
& \left.+\varepsilon \nabla_{x} \varrho \cdot \nabla_{x} \mathbf{u} \cdot \mathbf{V}-\varepsilon \nabla_{x} \varrho \cdot \nabla_{x}\left(\mathbf{u}_{\infty} \cdot \mathbf{V}\right)+\mathbb{S}\left(\nabla_{x} \mathbf{u}\right): \nabla_{x} \mathbf{V}\right) \mathrm{d} x \mathrm{~d} t
\end{aligned}
$$

for all $\tau$ in $\bar{I}$.

3. Employing in the regularized continuity equation (3.7) the test function $\varphi=-H^{\prime}(r)$, we get:

$$
\begin{aligned}
&-\int_{\Omega}\left(\varrho H_{\delta}^{\prime}(r)\right)(\tau, x) \mathrm{d} x+\int_{\Omega} \varrho_{0}(x) H_{\delta}^{\prime}(r(0, x) \mathrm{d} x \\
& \quad= \int_{0}^{\tau} \int_{\Omega}\left(-\varrho \frac{p_{\delta}^{\prime}(r)}{r}\left(\partial_{t} r+\mathbf{u} \cdot \nabla_{x} r\right)+\varepsilon H_{\delta}^{\prime \prime}(r) \nabla_{x} \varrho \cdot \nabla_{x} r\right) \mathrm{d} x \mathrm{~d} t \\
& \quad+\int_{0}^{\tau} \int_{\Gamma_{\mathrm{in}}} \varrho_{B} H_{\delta}^{\prime}(r) \mathbf{u}_{B} \cdot \mathbf{n} \mathrm{d} S_{x} \mathrm{~d} t+\int_{0}^{\tau} \int_{\Gamma_{\text {out }}} \varrho H_{\delta}^{\prime}(r) \mathbf{u}_{B} \cdot \mathbf{n} \varphi \mathrm{d} S_{x} \mathrm{~d} t
\end{aligned}
$$

with any $\tau \in \bar{I}$, where we have used the identity (2.5) in the form

$$
r H_{\delta}^{\prime}(r)-H(r)=p_{\delta}(r)
$$

cf. (3.5), (3.10).

4. The identity (5.5) yields

$$
\int_{\Omega}\left(r H_{\delta}^{\prime}(r)-H(r)\right) \mathrm{d} x-\int_{\Omega}\left(r H_{\delta}^{\prime}(r)-H_{\delta}(r)\right)(0, \cdot) \mathrm{d} x=\int_{0}^{\tau} \int_{\Omega} \partial_{t} p_{\delta}(r) \mathrm{d} x \mathrm{~d} t
$$

for all $\tau \in \bar{I}$.

5. Now, summing equations (5.2), (5.3), (5.4), (5.6) with the energy inequality (3.9) we get:

$$
\begin{aligned}
\int_{\Omega}( & \left.\frac{1}{2} \varrho|\mathbf{v}-\mathbf{V}|^{2}+E_{\delta}(\varrho \mid r)\right)(\tau, x) \mathrm{d} x \\
& +\int_{0}^{\tau} \int_{\Gamma_{\text {in }}} E_{\delta}\left(\varrho_{B} \mid \varrho\right)\left|\mathbf{u}_{B} \cdot \mathbf{n}\right| \mathrm{d} S_{x} \mathrm{~d} t+\int_{0}^{\tau} \int_{\Gamma_{\text {out }}}\left(H_{\delta}(\varrho)-\varrho H_{\delta}^{\prime}(r)\right) \mathbf{u}_{B} \cdot \mathbf{n} \mathrm{d} S_{x} \mathrm{~d} t \\
& +\int_{0}^{\tau} \int_{\Omega}\left(\mathbb{S}\left(\nabla_{x} \mathbf{u}\right): \nabla_{x}(\mathbf{v}-\mathbf{V})+\varepsilon H_{\delta}^{\prime \prime}(\varrho)\left|\nabla_{x} \varrho\right|^{2}+\varepsilon\left|\nabla_{x} \mathbf{V}\right|^{4}\right) \mathrm{d} x \mathrm{~d} t \\
\leq & \int_{\Omega}\left(\frac{1}{2} \varrho_{0}\left|\mathbf{u}_{0}-\mathbf{U}(0, \cdot)\right|^{2}+E_{\delta}\left(\varrho_{0} \mid r(0, \cdot)\right) \mathrm{d} x\right. \\
& +\int_{0}^{\tau} \int_{\Gamma_{\text {in }}}\left(-H_{\delta}\left(\varrho_{B}\right)+\varrho_{B} H_{\delta}^{\prime}(r)\right) \mathbf{u}_{B} \cdot \mathbf{n} \mathrm{d} S_{x} \mathrm{~d} t \\
& +\int_{0}^{\tau} \int_{\Omega}\left(\varrho(\mathbf{V}-\mathbf{v}) \cdot \partial_{t} \mathbf{V}+\varrho \mathbf{u} \cdot \nabla_{x} \mathbf{U} \cdot(\mathbf{V}-\mathbf{v})+\left(p_{\delta}(r)-p_{\delta}(\varrho)\right) \operatorname{div} \mathbf{U}\right.
\end{aligned}
$$




$$
\begin{array}{r}
\left.+\frac{r-\varrho}{r} p_{\delta}^{\prime}(r)\left(\partial_{t} r+\mathbf{u} \cdot \nabla_{x} r\right)-p_{\delta}^{\prime}(r) \mathbf{u} \cdot \nabla_{x} r-p_{\delta}(r) \operatorname{div} \mathbf{U}\right) \mathrm{d} x \mathrm{~d} t \\
+\int_{0}^{\tau} \int_{\Omega}\left(\mathbb{Z}_{\varepsilon}: \nabla_{x} \mathbf{V}+\varepsilon \nabla_{x} \varrho \cdot \nabla_{x}(\mathbf{u}-\mathbf{V}) \cdot \mathbf{V} \mathrm{d} x \mathrm{~d} t .\right.
\end{array}
$$

6. Now, we use in the before last line three elementary facts:

(a) First, in view of (2.12),

$$
\partial_{t} r+\mathbf{u} \cdot \nabla_{x} r=-r \operatorname{div} \mathbf{U}+(\mathbf{u}-\mathbf{U}) \cdot \nabla_{x} r
$$

(b) Second, by definition of $\mathbf{v}$ and $\mathbf{V}$,

$$
-\mathbf{u} \cdot \nabla_{x} r=-\mathbf{v} \cdot \nabla_{x} r-\mathbf{u}_{\infty} \cdot \nabla_{x} r,-\operatorname{div} \mathbf{U}=-\operatorname{div} \mathbf{V}-\operatorname{div} \mathbf{u}_{\infty} .
$$

(c) Third, by virtue of Stokes formula,

$$
-\int_{\Omega}\left(p_{\delta}^{\prime}(r) \mathbf{u}_{\infty} \cdot \nabla_{x} r+p_{\delta}(r) \operatorname{div} \mathbf{u}_{\infty}\right) \mathrm{d} x=-\int_{\Omega} p_{\delta}(r) \mathbf{u}_{B} \cdot \mathbf{n} \mathrm{d} x .
$$

(d) Consequently, the before last line reads

$$
\begin{aligned}
& \int_{0}^{\tau} \int_{\Omega}\left(\frac{r-\varrho}{r} p_{\delta}^{\prime}(r)\left(\partial_{t} r+\mathbf{u} \cdot \nabla_{x} r\right)-p_{\delta}^{\prime}(r) \mathbf{u} \cdot \nabla_{x} r-p_{\delta}(r) \operatorname{div} \mathbf{U}\right) \mathrm{d} x \mathrm{~d} t \\
& =\int_{0}^{\tau} \int_{\Omega}\left((\varrho-r) p_{\delta}^{\prime}(r) \operatorname{div} \mathbf{U}+\frac{r-\varrho}{r} p_{\delta}^{\prime}(r)(\mathbf{u}-\mathbf{U}) \cdot \nabla_{x} r-p_{\delta}^{\prime}(r) \mathbf{v} \cdot \nabla_{x} r-p_{\delta}(r) \operatorname{div} \mathbf{V}\right) \mathrm{d} x \mathrm{~d} t \\
& \quad-\int_{\Gamma_{\text {in }}} p_{\delta}(r) \mathbf{u}_{B} \cdot \mathbf{n} \mathrm{d} S-\int_{\Gamma_{\text {out }}} p_{\delta}(r) \mathbf{u}_{B} \cdot \mathbf{n} \mathrm{d} S .
\end{aligned}
$$

7. Inserting the latter formula into (5.7) and using also (3.10+3.11), (5.5), we obtain (5.1). Lemma 5.1 is thus proved.

\section{Limit $\varepsilon \rightarrow 0$. Proof of Theorem 2.2: start}

The aim in this section is to pass to the limit in the weak formulation (3.7 3.9) of the problem (3.13.4) $\left(_{\varepsilon}, \mathbf{u}_{\varepsilon}\right)$ in order to recover the weak formulation of problem (1.1] 1.5) - cf. (2.7 2.9) $\left.)_{p=p_{\delta}},(2.16)\right)_{p=p_{\delta}, H=H_{\delta}}$ and in the relative energy inequality (5.11) $\left(_{\varepsilon}, \mathbf{u}_{\varepsilon}, \mathbb{Z}_{\varepsilon}\right)$ to recover $(2.10){ }_{p=p_{\delta}, H=H_{\delta}, E=E_{\delta}}$.

Estimates (4.13 4.15) yield uniform bounds

$$
\begin{gathered}
\left\|\varrho_{\epsilon}\left|\mathbf{u}_{\epsilon}\right|^{2}\right\|_{L^{\infty}\left(I, L^{1}(\Omega)\right)} \leq L(\text { data }), \\
\left\|\mathbf{u}_{\epsilon}\right\|_{L^{2}\left(I, W^{1,2}(\Omega)\right)} \leq L(\text { data }),
\end{gathered}
$$




$$
\left\|\varrho_{\epsilon}\right\|_{L^{\infty}\left(I, L^{\gamma}(\Omega)\right)} \leq L(\text { data }) .
$$

Further, there is an $\delta$-dependent bound

$$
\delta^{1 / \beta}\left\|\varrho_{\epsilon}\right\|_{L^{\infty}\left(I, L^{\beta}(\Omega)\right)} \leq L(\text { data })
$$

and $\varepsilon$-dependent bounds

$$
\begin{aligned}
& \varepsilon^{1 / 4}\left\|\mathbf{u}_{\epsilon}-\mathbf{u}_{\infty}\right\|_{L^{4}\left(I, W^{1,4}(\Omega)\right)} \leq L(\text { data }, \delta),\left\|\mathbb{Z}_{\varepsilon}\right\|_{L^{4 / 3}\left(Q_{T}\right)} \rightarrow 0, \\
& \sqrt{\epsilon}\left\|\nabla \varrho_{\epsilon}\right\|_{L^{2}\left(Q_{T}\right)} \leq L(\text { data }) .
\end{aligned}
$$

Moreover, the momentum equation (3.8) provides a refined bound for the pressure, which reads

$$
\left\|\varrho_{\epsilon}\right\|_{L^{\beta+1}((0, T) \times K)} \leq L(\text { data, } \delta, K) \text {, with any compacts } K \subset \Omega,
$$

cf. [3, Lemma 5.2].

From estimates (6.3 6.4 ) we basically know, that there is a couple $(\varrho, \mathbf{u}) \in L^{\infty}\left((0, T) ; L^{\beta}(\Omega)\right) \times$ $L^{2}\left((0, T) ; W^{1,2}(\Omega)\right)$ which is a weak limit of a conveniently chosen subsequence of the sequence $\left(\varrho_{\varepsilon}, \mathbf{u}_{\varepsilon}\right)$ (not relabeled). It is proved in [3], that this limit belongs to the class (3.6) and satisfies the continuity equation (2.8), the momentum equation (2.9) $p_{p=p_{\delta}}$ and the energy inequality $(2.16){ }_{p=p_{\delta}, H=H_{\delta}}$. This nontrivial result is obtained through the key idea asserting that

$$
\varrho_{\varepsilon} \rightarrow \varrho \text { a.e. in } Q_{T}{ }^{7}
$$

It remains to pass to the limit in the approximate relative energy inequality (5.11) ${\left(\varrho_{\varepsilon}, \mathbf{u}_{\varepsilon}, \mathbb{Z}_{\varepsilon}\right)}$ and get (2.10) $p_{p=p_{\delta}, E=E_{\delta}, H=H_{\delta}}$. To this end we take $0<s<t<T$ and integrate (5.11) ${\left(\varrho_{\varepsilon}, \mathbf{u}_{\varepsilon}, \mathbb{Z}_{\varepsilon}\right)}$ over $\tau$ from $s$ to $t$. We get

$$
\begin{aligned}
& \int_{s}^{t} \int_{\Omega}\left(\frac{1}{2} \varrho_{\varepsilon}\left|\mathbf{v}_{\varepsilon}-\mathbf{V}\right|^{2}+E_{\delta}\left(\varrho_{\varepsilon} \mid r\right)\right)(\tau, x) \mathrm{d} x \mathrm{~d} \tau \\
& \quad+\int_{s}^{t} \int_{0}^{\tau} \int_{\Gamma_{\mathrm{in}}} E_{\delta}\left(\varrho_{B} \mid \varrho_{\varepsilon}\right)\left|\mathbf{u}_{B} \cdot \mathbf{n}\right| \mathrm{d} S_{x} \mathrm{~d} t \mathrm{~d} \tau+\int_{s}^{t} \int_{0}^{\tau} \int_{\Gamma_{\text {out }}} E_{\delta}\left(\varrho_{\varepsilon} \mid r\right) \mathbf{u}_{B} \cdot \mathbf{n} \mathrm{d} S_{x} \mathrm{~d} t \mathrm{~d} \tau \\
& \quad+\int_{s}^{t} \int_{0}^{\tau} \int_{\Omega}\left(\mathbb{S}\left(\nabla_{x} \mathbf{u}_{\varepsilon}\right): \nabla_{x}\left(\mathbf{v}_{\varepsilon}-\mathbf{V}\right)+\varepsilon H_{\delta}^{\prime \prime}\left(\varrho_{\varepsilon}\right)\left|\nabla_{x} \varrho_{\varepsilon}\right|^{2}+\varepsilon\left|\nabla_{x} \mathbf{v}_{\varepsilon}\right|^{4}\right) \mathrm{d} x \mathrm{~d} t \mathrm{~d} \tau \\
& \leq(t-s) \int_{\Omega}\left(\frac{1}{2} \varrho_{0}\left|\mathbf{v}_{0}-\mathbf{V}(0, \cdot)\right|^{2}+E_{\delta}\left(\varrho_{0} \mid r(0, \cdot)\right) \mathrm{d} x\right. \\
& \quad+(t-s) \int_{0}^{\tau} \int_{\Gamma_{\text {in }}}\left(H_{\delta}(r)-r H_{\delta}^{\prime}(r)-H_{\delta}\left(\varrho_{B}\right)+\varrho_{B} H_{\delta}^{\prime}(r)\right) \mathbf{u}_{B} \cdot \mathbf{n d} S_{x} \mathrm{~d} t \mathrm{~d} \tau \\
& \quad+\int_{t}^{s} \int_{0}^{\tau} \int_{\Omega}\left(\varrho_{\varepsilon}\left(\mathbf{V}-\mathbf{v}_{\varepsilon}\right) \cdot \partial_{t} \mathbf{V}+\varrho_{\varepsilon} \mathbf{u}_{\varepsilon} \cdot \nabla_{x} \mathbf{U} \cdot\left(\mathbf{V}-\mathbf{v}_{\varepsilon}\right)\right.
\end{aligned}
$$

\footnotetext{
${ }^{7}$ This is the key point in the existence theory of compressible Navier-Stokes equations, whatever is the geometrical setting and whatever are the boundary conditions, cf. Lions [22, [15].
} 


$$
\begin{aligned}
& +\left(p_{\delta}(r)-p_{\delta}^{\prime}(r)\left(r-\varrho_{\varepsilon}\right)-p_{\delta}\left(\varrho_{\varepsilon}\right)\right) \operatorname{div} \mathbf{U} \\
& \left.\quad+\frac{r-\varrho}{r} p_{\delta}^{\prime}(r)\left(\mathbf{v}_{\varepsilon}-\mathbf{V}\right) \cdot \nabla_{x} r-p_{\delta}^{\prime}(r) \mathbf{v}_{\varepsilon} \cdot \nabla_{x} r-p_{\delta}(r) \operatorname{div} \mathbf{V}\right) \mathrm{d} x \mathrm{~d} t \mathrm{~d} \tau \\
& +\int_{t}^{s} \int_{0}^{\tau} \int_{\Omega}\left(\mathbb{Z}_{\varepsilon}: \nabla_{x} \mathbf{V}+\varepsilon \nabla_{x} \varrho_{\varepsilon} \cdot \nabla_{x}\left(\mathbf{u}_{\varepsilon}-\mathbf{V}\right) \cdot \mathbf{V}\right) \mathrm{d} x \mathrm{~d} t \mathrm{~d} \tau .
\end{aligned}
$$

The relation (6.8) in combination with estimates (6.1) 6.7) employed in (6.9) allows to pass to the limit in all of its terms except the term

$$
-\int_{t}^{s} \int_{0}^{\tau} \int_{\Omega} p_{\delta}\left(\varrho_{\varepsilon}\right) \operatorname{div} \mathbf{U} \mathrm{d} x \mathrm{~d} t \mathrm{~d} \tau
$$

Indeed:

1. Under the assumption $p^{\prime}(\varrho)>0$ the Helmholtz function $H_{\delta}$ is strictly convex. We can therefore omit at the left hand side of (5.1) all integrals over the boundary, since they are non negative. The same is true for the right hand side volumic integrals containing $\varepsilon$ as a multiplier.

2. We use lower weak semicontinuity of the convex functionals together with standard compactness argument: 8 in the remaining terms of the left hand side of (6.9).

3. We employ estimate (6.6) to get rid of the term containing multiplication by $\varepsilon$ at the right hand side, and relation (6.5) to get rid of the term containing $\mathbb{Z}_{\varepsilon}$.

4. We use the standard compactness arguments in all terms of the right hand side except the term (6.10). The term (6.10) cannot be so far treated, due to the fact that estimate (6.7) does not hold up to the boundary.

To remedy to the above problem, we intend to prove that the integral of the pressure over the spacetime cylinder whose basis is an inner neighborhood of the boundary $\partial \Omega$, is comparable with the measure of the neighborhood in a certain positive power. To this end, we show first the following lemma.

Lemma 6.1. Let $\Omega$ be a bounded Lipschitz domain, $\hat{U}_{h}^{-}, h>0$ an inner neighborhood of its boundary see (2.6) - and $\alpha, \kappa>1$. Consider a sequence $\left(p_{\varepsilon}, \mathbf{z}_{\varepsilon}, \mathbf{F}_{\varepsilon}, \mathbb{G}_{\varepsilon}\right)_{\varepsilon>0}$ of functions which satisfy equation

$$
\partial_{t} \mathbf{z}_{\varepsilon}+\mathbf{F}_{\varepsilon}+\operatorname{div} \mathbb{G}_{\varepsilon}+\nabla_{x} p_{\varepsilon}=0 \text { in } \mathcal{D}^{\prime}\left(Q_{T} ; \mathbb{R}^{d}\right)
$$

Suppose finally that $p_{\varepsilon} \in L^{1}\left(Q_{T}\right)$, while

$$
\left(z_{\varepsilon}, \mathbf{F}_{\varepsilon}, \mathbb{G}_{\varepsilon}\right)_{\varepsilon>0} \text { is bounded in } L^{\infty}\left(0, T ; L^{\alpha}(\Omega)\right) \times L^{\kappa}\left(Q_{T}\right) \times L^{\kappa}\left(Q_{T}\right) \text { by } k>0
$$

uniformly with respect to $\varepsilon$.

\footnotetext{
${ }^{8}$ Here and hereafter, the standard compactness arguments include the Sobolev imbeddings, Arzela-Ascoli theorem and Lions-Aubin lemma.
} 
Then there exists $h_{0}>0$ and $c=c(k, T, \Omega)>0$ such that

$$
\int_{0}^{T} \int_{\hat{U}_{h}^{-}} p\left(\varrho_{\varepsilon}\right) \mathrm{d} x \mathrm{~d} t \leq \operatorname{ch}^{\Gamma}, \text { where } \Gamma=\min \left\{1 / \alpha^{\prime}, 1 / \kappa^{\prime}\right\},
$$

for all $0<h<h_{0}$ uniformly with respect to $\varepsilon$.

\section{Proof of Lemma 6.1}

We shall proceed in several steps.

1. We shall start the proof by recalling the so called Bogovskii lemma, see e.g. Galdi [20] or [24].

Lemma 6.2. Let $\Omega$ be a bounded Lipschitz domain. Then there exists a linear operator

$$
\left.\mathcal{B}:\left\{f \in C_{c}^{\infty}\left(\Omega ; \mathbb{R}^{3}\right) \mid \int_{\Omega} f \mathrm{~d} x=0\right\} \mapsto C_{c}^{\infty}\left(\Omega ; \mathbb{R}^{3}\right)\right\}
$$

such that:

(a) $\operatorname{div} \mathcal{B}[f]=f$

(b) $\mathcal{B}$ is bounded linear operator from $\bar{L}^{p}(\Omega)$ to $W^{1, p}(\Omega)$ for any $1<p<\infty$ (i.e. there is $c=c(p)>0$ such that $\|\mathcal{B}[f]\|_{W^{1, p}\left(\Omega ; \mathbb{R}^{3}\right)} \leq c(p)\|f\|_{L^{p}(\Omega)}$ for all $\left.f \in \bar{L}^{p}(\Omega)\right)$. In the above $\bar{L}^{p}(\Omega):=\left\{f \in L^{p}(\Omega) \mid \int_{\Omega} f \mathrm{~d} x=0\right\}$.

2. Let $h_{0}$ be sufficiently small, such that $\hat{U}_{h}^{-} \subset \Omega$ and $\left|\hat{U}_{h}^{-}\right|<|\Omega| / 2$. We take in Lemma 6.2

$$
f(x)=1_{\Omega \backslash \hat{U}_{h}^{-}}(x)-\frac{|\Omega|-\left|\hat{U}_{h}^{-}\right|}{|\Omega|},
$$

where $1_{A}$ denotes the characteristic function of the set $A$. We observe that: 1$)$ Since $\Omega$ is Lipschitz, there exists $\bar{c}>0$ such that for all $0<h<h_{0}$,

$$
\left|\hat{U}_{h}^{-}\right| \leq \bar{c} h
$$

2) Function $f$ satisfies all assumptions of Lemma 6.2 with any $1<p<\infty$. Consequently, setting $\psi(x)=\mathcal{B}[f]$, we get estimate

$$
\|\psi\|_{W^{1, p}(\Omega)} \leq c(p) h^{1 / p} \text { for all } 0<h<h_{0}
$$

3. Now, we use in equation (6.11) as the test function the function $\varphi(t, x)=\eta(t) \psi(x)$, where $0 \leq \eta \in C_{c}^{1}((0, T)), \eta(t)=1$ in $(\Delta, T-\Delta), \Delta \in(0, T / 2),\left|\partial_{t} \eta\right| \leq 2 / \Delta$. 
(This is an admissible test function, as one can show by a density argument.) We get

$$
-\int_{0}^{T} \eta \int_{\Omega} p \operatorname{div} \varphi \mathrm{d} x \mathrm{~d} t=I_{1}+I_{2}+I_{3}
$$

where

$$
I_{1}=\int_{0}^{T} \partial_{t} \eta \int_{\Omega} \mathbf{z}_{\varepsilon} \cdot \psi \mathrm{d} x \mathrm{~d} t, I_{2}=\int_{0}^{T} \eta \int_{\Omega} \mathbb{G}_{\varepsilon}: \nabla_{x} \psi \mathrm{d} x \mathrm{~d} t, I_{3}=-\int_{0}^{T} \eta \int_{\Omega} \mathbf{F}_{\varepsilon} \cdot \psi \mathrm{d} x \mathrm{~d} t .
$$

Seeing that

$$
-\int_{0}^{T} \eta \int_{\Omega} p \operatorname{div} \varphi \mathrm{d} x \mathrm{~d} t=\frac{|\Omega|-\left|\hat{U}_{h}^{-}\right|}{|\Omega|} \int_{0}^{T} \eta \int_{\hat{U}_{h}^{-}} p\left(\varrho_{\varepsilon}\right) \mathrm{d} x \mathrm{~d} t-\frac{\left|\hat{U}_{h}^{-}\right|}{|\Omega|} \int_{\Omega \backslash \hat{U}_{h}^{-}} p\left(\varrho_{\varepsilon}\right) \mathrm{d} x \mathrm{~d} t
$$

and that

$$
\left|I_{1}\right| \leq c\left\|\mathbf{z}_{\varepsilon}\right\|_{L^{\infty}\left(0, T ; L^{\alpha}(\Omega)\right)}\|\psi\|_{L^{\alpha^{\prime}}(\Omega)},\left|I_{2}\right|+\left|I_{3}\right| \leq c\left\|\mathbf{F}_{\varepsilon}, \mathbb{G}_{\varepsilon}\right\|_{L^{\kappa}\left(Q_{T}\right)}\|\psi\|_{L^{\kappa^{\prime}}(\Omega)}
$$

with $c$ independent of $\eta$, i.e., in particular, independent of $\Delta$. Inserting both latter observations to (6.15) while taking into account (6.13) and (6.14) completes the proof of Lemma 6.1.

We are now ready to treat the problematic term (6.10). We apply Lemma 6.1 to the momentum equation (3.8), i.e., we set $p_{\varepsilon}=p_{\delta}\left(\varrho_{\varepsilon}\right), \mathbf{z}_{\varepsilon}=\varrho_{\varepsilon} \mathbf{v}_{\varepsilon}, \mathbf{F}_{\varepsilon}=\varrho_{\varepsilon} \mathbf{u}_{\varepsilon} \cdot \nabla_{x} \mathbf{u}_{\infty}+\varepsilon \nabla_{x} \varrho_{\varepsilon} \cdot \nabla_{x} \mathbf{v}_{\varepsilon}, \mathbb{G}_{\varepsilon}=-\mathbb{Z}_{\varepsilon}+$ $\varrho_{\varepsilon} \mathbf{u}_{\varepsilon} \otimes \mathbf{v}_{\varepsilon}-\mathbb{S}\left(\nabla_{x} \mathbf{u}_{\varepsilon}\right)$. Due to (6.1), (6.4), $\mathbf{z}_{\varepsilon}$ is bounded in $L^{\infty}\left(0, T ; L^{\frac{2 \beta}{\beta+1}}(\Omega)\right)$, by virtue of (6.1), (6.2), (6.4), (6.5), $\mathbb{G}_{\varepsilon}$ is bounded in $L^{\min \left\{4 / 3, \frac{6 \beta}{4 \beta+3}\right\}}\left(Q_{T}\right)$, and finally, due to (6.1), (6.4), (6.5) and (6.6), $\mathbf{F}_{\varepsilon}$ is bounded in $L^{4 / 3}\left(Q_{T}\right)$. We thus obtain

$$
\limsup _{\varepsilon \rightarrow 0}\left|\int_{t}^{s} \int_{0}^{\tau} \int_{\hat{U}_{h}^{-}} p_{\delta}\left(\varrho_{\varepsilon}\right) \operatorname{div} \mathbf{U} \mathrm{d} x \mathrm{~d} t \mathrm{~d} \tau\right| \leq c \sup _{(t, x) \in Q_{T}}|\operatorname{div} \mathbf{U}(t, x)| h^{\Gamma} \text { with some } \Gamma>0,
$$

for all $0<h<h_{0}$ and $0 \leq t<s \leq T$.

We may write with any $0<h<h_{0}$,

$$
-\int_{t}^{s} \int_{0}^{\tau} \int_{\Omega} p_{\delta}\left(\varrho_{\varepsilon}\right) \operatorname{div} \mathbf{U} \mathrm{d} x \mathrm{~d} t \mathrm{~d} \tau=\int_{t}^{s} \int_{0}^{\tau} \int_{\hat{U}_{h}^{-}} p_{\delta}\left(\varrho_{\varepsilon}\right) \operatorname{div} \mathbf{U} \mathrm{d} x \mathrm{~d} t \mathrm{~d} \tau+\int_{t}^{s} \int_{0}^{\tau} \int_{\Omega \backslash \hat{U}_{h}^{-}} p_{\delta}\left(\varrho_{\varepsilon}\right) \operatorname{div} \mathbf{U} \mathrm{d} x \mathrm{~d} t \mathrm{~d} \tau,
$$

where due to (6.7) and (6.8),

$$
\lim _{\varepsilon \rightarrow 0} \int_{t}^{s} \int_{0}^{\tau} \int_{\Omega \backslash \hat{U}_{h}^{-}} p_{\delta}\left(\varrho_{\varepsilon}\right) \operatorname{div} \mathbf{U} \mathrm{d} x \mathrm{~d} t \mathrm{~d} \tau=\int_{t}^{s} \int_{0}^{\tau} \int_{\Omega \backslash \hat{U}_{h}^{-}} p_{\delta}(\varrho) \operatorname{div} \mathbf{U} \mathrm{d} x \mathrm{~d} t \mathrm{~d} \tau
$$

with any $0<h<h_{0}$. We already know that, in particular, $p(\varrho) \in L^{1}\left(Q_{T}\right)$. Therefore

$$
\lim _{h \rightarrow 0} \int_{t}^{s} \int_{0}^{\tau} \int_{\Omega \backslash \hat{U}_{h}^{-}} p_{\delta}(\varrho) \operatorname{div} \mathbf{U} \mathrm{d} x \mathrm{~d} t \mathrm{~d} \tau=\int_{t}^{s} \int_{0}^{\tau} \int_{\Omega} p_{\delta}(\varrho) \operatorname{div} \mathbf{U} \mathrm{d} x \mathrm{~d} t \mathrm{~d} \tau .
$$


Using the both latter facts and estimate (6.16) in the decomposition (6.17) we get the desired conclusion, namely, that

$$
\int_{t}^{s} \int_{0}^{\tau} \int_{\Omega} p_{\delta}\left(\varrho_{\varepsilon}\right) \operatorname{div} \mathbf{U} \mathrm{d} x \mathrm{~d} t \mathrm{~d} \tau \rightarrow \int_{t}^{s} \int_{0}^{\tau} \int_{\Omega} p_{\delta}(\varrho) \operatorname{div} \mathbf{U} \mathrm{d} x \mathrm{~d} t \mathrm{~d} \tau
$$

as $\varepsilon \rightarrow 0$. This is the last element needed to get from inequality (6.9),

$$
\begin{aligned}
& \int_{s}^{t} \int_{\Omega}\left(\frac{1}{2} \varrho|\mathbf{v}-\mathbf{V}|^{2}+E_{\delta}(\varrho \mid r)\right)(\tau, x) \mathrm{d} x \mathrm{~d} \tau \\
& \leq(t-s) \int_{\Omega}\left(\frac{1}{2} \varrho_{0}\left|\mathbf{v}_{0}-\mathbf{V}(0, \cdot)\right|^{2}+E_{\delta}\left(\varrho_{0} \mid r(0, \cdot)\right) \mathrm{d} x\right. \\
& \quad+(t-s) \int_{0}^{\tau} \int_{\Gamma_{\mathrm{in}}}\left(H_{\delta}(r)-r H_{\delta}^{\prime}(r)-H_{\delta}\left(\varrho_{B}\right)+\varrho_{B} H_{\delta}^{\prime}(r)\right) \mathbf{u}_{B} \cdot \mathbf{n} \mathrm{d} S_{x} \mathrm{~d} t \mathrm{~d} \tau \\
& \quad+\int_{t}^{s} \int_{0}^{\tau} \int_{\Omega}\left(\varrho(\mathbf{V}-\mathbf{v}) \cdot \partial_{t} \mathbf{V}+\varrho \mathbf{u} \cdot \nabla_{x} \mathbf{U} \cdot(\mathbf{V}-\mathbf{v})\right. \\
& \quad+\left(p_{\delta}(r)-p_{\delta}^{\prime}(r)(r-\varrho)-p_{\delta}(\varrho)\right) \operatorname{div} \mathbf{U} \\
& \left.\quad+\frac{r-\varrho}{r} p_{\delta}^{\prime}(r)(\mathbf{v}-\mathbf{V}) \cdot \nabla_{x} r-p_{\delta}^{\prime}(r) \mathbf{v} \cdot \nabla_{x} r-p_{\delta}(r) \operatorname{div} \mathbf{V}\right) \mathrm{d} x \mathrm{~d} t \mathrm{~d} \tau
\end{aligned}
$$

for all $0 \leq s<t \leq T$. The final step is to devide (6.18) by $t-s$ and effectuate the limit $t \rightarrow s$. The theorem on Lebesgue points then guarantees the satisfaction of the relative energy inequality (2.10) with $(p, H, E)$ replaced by $\left(p_{\delta}, H_{\delta}, E_{\delta}\right)$.

\section{Limit $\delta \rightarrow 0$. Proof of Theorem 2.2: end}

Our final goal is to pass to the limit in the weak formulation (1.1 1.5) $)_{p=p_{\delta}, \varrho_{\delta}, \mathbf{u}_{\delta}-\text { cf. }(2.7-2.9)} p_{p=p_{\delta}, \varrho_{\delta}, \mathbf{u}_{\delta}}$, $(2.16)_{p=p_{\delta}, H=H_{\delta}, \varrho_{\delta}, \mathbf{u}_{\delta}}$, in order to recover the weak formulation (2.7 2.9), (2.16) of problem (1.1 1.5) and in the relative energy inequality (2.10) ${ }_{p=p_{\delta}, H=H_{\delta}, E=E_{\delta}, \varrho_{\delta}, \mathbf{u}_{\delta}}$ in order to recover (2.10).

Estimates (6.1 6.4) yield uniform bounds

$$
\begin{gathered}
\left\|\varrho_{\delta} \mathbf{u}_{\delta}^{2}\right\|_{L^{\infty}\left(I, L^{1}(\Omega)\right)} \leq L(\text { data }) \\
\left\|\mathbf{u}_{\delta}\right\|_{L^{2}\left(I, W^{1,2}\left(\Omega ; R^{3}\right)\right)} \leq L(\text { data })
\end{gathered}
$$

and $\delta$ dependent bound

$$
\delta^{1 / \beta}\left\|\varrho_{\delta}\right\|_{L^{\infty}\left(I, L^{\beta}(\Omega)\right)} \leq L(\text { data }) .
$$

Moreover, the momentum equation provides, under condition $\gamma>3 / 2$, a refined bound for pressure, which reads

$$
\left\|\varrho_{\delta}\right\|_{L^{\gamma+\alpha(\gamma)}((0, T) \times K)} \leq L(\text { data, } K) \text {, with any compacts } K \subset \Omega,
$$

and with $\alpha=\min \left\{\frac{2}{3} \gamma-1, \frac{\gamma}{2}\right\}$, cf. [3, Lemma 6.2]. 
From estimates (6.3 6.4) we deduce, that there is a couple $(\varrho, \mathbf{u}) \in L^{\infty}\left((0, T) ; L^{\gamma}(\Omega)\right) \times L^{2}\left((0, T) ; W^{1,2}(\Omega)\right)$ which is a weak limit of a conveniently chosen subsequence of the sequence $\left(\varrho_{\delta}, \mathbf{u}_{\delta}\right)$ (not relabeled). We also know that

$$
\varrho_{\delta} \rightarrow \varrho \text { a.e. in } Q_{T}
$$

provided $\gamma>3 / 2$. As underlined in the previous section, the latter convergence relation is crucial in the theory of compressible Navier-Stokes equations and its proof is quite involved. We refer to [3, Section 6] for the proof in the present context. It is shown, that this limit belongs to the class (2.7) and satisfies the continuity equation (2.8), the momentum equation (2.9) and the energy inequality (2.16). Our task is

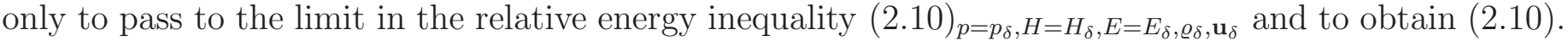

Reasoning as in the previous section we discover that the only problematic term is

$\int_{0}^{\tau} \int_{\Omega} p_{\delta}\left(\varrho_{\delta}\right) \operatorname{div} \mathbf{U} \mathrm{d} x \mathrm{~d} t$. Indeed, since estimate (7.4) is only local, it is not clear $p_{\delta}\left(\varrho_{\delta}\right)$ converges weakly to $p_{\delta}(\varrho)$ "near the boundary", even if we know (7.5). We will treat this difficulty exactly in the same manner as we have treated the term (6.10) in the previous section, by using Lemma 6.1. This lemma will be applied to the momentum equation $(\underline{2.9})_{p=p_{\delta}, \varrho_{\delta}, \mathbf{u}_{\delta}}$ yielding estimate (6.16) with $\varrho_{\varepsilon}$ replaced by $\varrho_{\delta}$. In this calculation, again, the value $3 / 2$ is a threshold that cannot be achieved.

The rest of the reasoning is the same as in the previous section and thus left to the reader. Theorem 2.2 is proved.

\section{Stability and weak-strong uniqueness: Proof of Theorem 2.4}

In this section we shall prove Theorem 2.4. We shall show that the strong solutions to the problem (1.1-1.5) are stable in the class of dissiptive weak solutions. In particular, any dissipative weak solution of the problem (1.1 1.5) coincides with the strong solution of the same problem emanating from the same initial data and the same boundary conditions.

\subsection{Relative energy inequality with a strong solution as a test function}

If the test functions $(r, \mathbf{U})$ in the relative energy inequality (2.10) obey equations (1.1, 1.2) almost everywhere in $Q_{T}$ the right hand side of the relative energy becomes quadratic in differences $(\varrho-r, \mathbf{u}-\mathbf{U})$. This observation is subject of the following lemma:

Lemma 8.1. Let $\Omega$ be a bounded Lipschitz domain. Suppose that the pressure satisfies assumptions (2.2) 9 Let $(\varrho, \mathbf{u})$ be a dissipative weak solution to the Navier-Stokes equations (1.1-1.5) emanating from the finite energy initial data $\left(\varrho_{0}, \mathbf{u}_{0}\right)$ in class (2.19) and boundary data $\left(\varrho_{B}, \mathbf{u}_{B}\right)$ in class (2.1), corresponding to extension $\mathbf{u}_{\infty}$ of $\mathbf{u}_{B^{-}} c f$. (2.13). Let $(r, \mathbf{U})$ belonging to the class

$$
0<\underline{r} \leq r \leq \bar{r}<\infty ; \quad \mathbf{U} \in L^{\infty}((0, T) \times \Omega)
$$

\footnotetext{
${ }^{9}$ This Lemma holds without condition $p(0)=0, p^{\prime}(\varrho)>0$; regularity assumption of $(2.2)$ is enough.
} 


$$
\partial_{t} r, \partial_{t} \mathbf{U}, \nabla_{x} r, \nabla_{x} \mathbf{U} \in L^{2}(0, T ; C(\bar{\Omega}))
$$

be a strong solution of the same equations with initial data $(r(0), \mathbf{U}(0))=\left(r_{0}, \mathbf{U}_{0}\right)$ and boundary data $\left(\left.r\right|_{\Gamma_{\text {in }}},\left.\mathbf{U}\right|_{\partial \Omega}\right)=\left(r_{B}, \mathbf{u}_{B}\right)$. Then the relative energy inequality (2.10) takes the form:

$$
\begin{gathered}
\int_{\Omega}\left(\frac{1}{2} \varrho|\mathbf{v}-\mathbf{V}|^{2}+E(\varrho \mid r)\right)(\tau) \mathrm{d} x+\int_{0}^{\tau} \int_{\Omega} \mathbb{S}\left(\nabla_{x}(\mathbf{v}-\mathbf{V})\right): \nabla_{x}(\mathbf{v}-\mathbf{V}) \mathrm{d} x \mathrm{~d} t \\
\leq \int_{\Omega}\left(\frac{1}{2} \varrho_{0}\left|\mathbf{v}_{0}-\mathbf{V}_{0}\right|^{2}+E\left(\varrho_{0} \mid r_{0}\right)\right) \mathrm{d} x+\mathcal{R}(\varrho, \mathbf{v} \mid r, \mathbf{V}),
\end{gathered}
$$

for a.e. $\tau \in I$. In the above, the remainder reads

$$
\begin{gathered}
\mathcal{R}(\varrho, \mathbf{v} \mid r, \mathbf{V})=\int_{0}^{\tau} \int_{\Gamma_{\mathrm{in}}}\left(H\left(r_{B}\right)-H\left(\varrho_{B}\right)+\left(\varrho_{B}-r_{B}\right) H^{\prime}\left(r_{B}\right)\right) \mathbf{u}_{B} \cdot \mathbf{n} \mathrm{d} S \mathrm{~d} t \\
\left.+\int_{0}^{\tau} \int_{\Omega}(\varrho-r)(\mathbf{V}-\mathbf{v}) \cdot \partial_{t} \mathbf{U}+(\varrho-r) \mathbf{U} \cdot \nabla_{x} \mathbf{U} \cdot(\mathbf{V}-\mathbf{v})+\varrho(\mathbf{v}-\mathbf{V}) \cdot \nabla_{x} \mathbf{U} \cdot(\mathbf{V}-\mathbf{v})\right) \mathrm{d} x \mathrm{~d} t \\
+\int_{0}^{\tau} \int_{\Omega}\left(p(r)-p^{\prime}(r)(r-\varrho)-p(\varrho)\right) \operatorname{div} \mathbf{U} \mathrm{d} x \mathrm{~d} t+\int_{0}^{\tau} \int_{\Omega}\left(\left(1-\frac{\varrho}{r}\right) p^{\prime}(r)(\mathbf{v}-\mathbf{V}) \cdot \nabla_{x} r\right) \mathrm{d} x \mathrm{~d} t
\end{gathered}
$$

and $\mathbf{v}=\mathbf{u}-\mathbf{u}_{\infty}, \mathbf{v}_{0}=\mathbf{u}_{0}-\mathbf{u}_{\infty}, \mathbf{V}=\mathbf{U}-\mathbf{u}_{\infty}, \mathbf{V}_{0}=\mathbf{U}_{0}-\mathbf{u}_{\infty}$.

\section{Proof of Lemma 8.1}

We start by the observation that due to the regularity (8.1) the couple $(r, \mathbf{V})$ satisfies

$$
\begin{gathered}
\partial_{t} r+\operatorname{div}(r \mathbf{U})=0 \text { a.e. in }(0, T) \times \Omega, \\
r \partial_{t} \mathbf{U}+r \mathbf{U} \cdot \nabla \mathbf{U}+\nabla p(r)=\operatorname{div} \mathbb{S}(\nabla \mathbf{U}) \text { a.e. in }(0, T) \times \Omega .
\end{gathered}
$$

Multiplying (8.4) scalarly by $\mathbf{v}-\mathbf{V}$ and integrating over $\Omega$, we get

$$
\int_{\Omega}\left(r \partial_{t} \mathbf{U}+r \mathbf{U} \cdot \nabla \mathbf{U}+\nabla p(r)\right) \cdot(\mathbf{v}-\mathbf{V}) \mathrm{d} x \mathrm{~d} t+\int_{\Omega} \mathbb{S}(\nabla \mathbf{U}): \nabla(\mathbf{v}-\mathbf{V}) \mathrm{d} x \mathrm{~d} t=0,
$$

where we have used the integration by parts in the last integral.

Next we calculate

$$
\int_{\Omega} \nabla_{x} p(r) \cdot(\mathbf{v}-\mathbf{V}) \mathrm{d} x=\int_{\Omega} p^{\prime}(r) \mathbf{v} \cdot \nabla_{x} r \mathrm{~d} x+\int_{\Omega} p(r) \operatorname{div} \mathbf{V} \mathrm{d} x .
$$

Adding (8.4) to the inequality (2.10) while taking into acount the above identity and relations (3.10) between $p$ and $H$, we obtain the inequality (8.2). Lemma 8.1 is proved. 


\subsection{Two algebraic relations}

It is evident that under the assumption $p^{\prime}(\varrho)>0$, the Helmholtz function $H$ is strictly convex and therefore $E(\varrho \mid r)>0$ for all $\varrho \geq 0, r>0$, and $E(\varrho \mid r)=0$ if and only if $\varrho=r$. We can, however, prove more:

Lemma 8.2. Let $0<a<b<\infty$ and let $p$ satisfies (2.2). Then there exists a number $c=c(a, b)>0$ such that for all $\varrho \in[0, \infty)$ and $r \in[a, b]$,

$$
E(\varrho \mid r) \geq c(a, b)\left(1_{\mathcal{O}_{\text {res }}}(\varrho)+\varrho 1_{\mathcal{O}_{\text {res }}}(\varrho)+(\varrho-r)^{2} 1_{\mathcal{O}_{\text {ess }}}(\varrho)\right)
$$

where $E$ is defined in 2.4) and

$$
\mathcal{O}_{\text {ess }}=[a / 2,2 b], \mathcal{O}_{\text {res }}=[0, \infty) \backslash \mathcal{O}_{\text {ess }} .
$$

\section{Proof}

If $\varrho \in[a / 2,2 b]$ we use the strict convexity of $H$ to obtain that

$$
E(\varrho \mid r) \geq c|\varrho-r|^{2} \text { where } c=c(a, b)>0 .
$$

If $\varrho \in R_{+} \backslash[a / 2,2 b]$, we observe that

$$
\partial_{\varrho} E(\varrho \mid r)=H^{\prime}(\varrho)-H^{\prime}(r), \quad \partial_{r} E(\varrho \mid r)=H^{\prime \prime}(r)(r-\varrho),
$$

where $s \rightarrow H^{\prime}(s)$ is an increasing function on $(0, \infty)$. Now, relying on the monotonicity of functions $s \rightarrow E(s \mid r)$ and $s \rightarrow E(\varrho \mid s)$ induced by the above formulas, we consider two situations. 1) If $\varrho>2 b$, we observe that $E(\varrho \mid 2 b)>0$, whence $H(\varrho)+p(2 b)>H^{\prime}(2 b) \varrho$. Consequently,

$$
p(2 b)-p(b)+E(\varrho \mid r) \geq p(2 b)-p(b)+E(\varrho \mid b)=H(\varrho)+p(2 b)-H^{\prime}(b) \varrho \geq\left(H^{\prime}(2 b)-H^{\prime}(b)\right) \varrho .
$$

This inequality and the fact that $E(\varrho, r) \geq E(2 b, b)>0, p(2 b)>p(b), H^{\prime}(2 b)>H^{\prime}(b)$ yield

$$
E(\varrho \mid r) \geq c(1+\varrho)
$$

with some $c=c(b)>0.2)$ If $\varrho<a / 2$ then

$$
E(\varrho \mid r) \geq E(a / 2 \mid a) \geq \frac{E(a / 2 \mid a)}{a} \varrho+\frac{E(a / 2 \mid a)}{2} \geq c(1+\varrho)
$$

with some $c=c(a)>0$. Lemma 8.2 is proved.

Since $\varrho \mapsto p(\varrho)$ is bounded on any compact subset of $[0, \infty)$ and since according to the definition of $E(\cdot \mid \cdot), H(\varrho) \leq c(E(\varrho \mid r)+1+\varrho)$ with any $\varrho \in[0, \infty)$ and any $r \in[a / 2,2 b]$, where $c=c(a, b)>0$, we deduce from estimate (8.6) the following result:

Corollary 8.3. Suppose that $p$ assumption (2.2) and (2.20). Then for any $\varrho \in[0, \infty)$ and $r \in[a / 2,2 b]$ we have, in addition to inequality (8.6),

$$
p(\varrho) 1_{\mathcal{O}_{\text {res }}}(\varrho) \leq c E(\varrho \mid r)
$$

with some $c=c(a, b)>0$. 


\subsection{Proof of Theorem 2.4}

\subsubsection{The Gronwall inequality}

The goal now is to find an estimate of the left hand side of (8.2) from below by

$$
c \int_{0}^{\tau}\|\mathbf{v}-\mathbf{V}\|_{W^{1,2}\left(\Omega ; \mathbb{R}^{3}\right)}^{2} \mathrm{~d} t-\bar{c}^{\prime} \int_{0}^{\tau} \mathcal{E}(\varrho, \mathbf{v} \mid r, \mathbf{V}) \mathrm{d} t+\left.\mathcal{E}(\varrho, \mathbf{v} \mid r, \mathbf{V})\right|_{0} ^{\tau},
$$

and the right hand side from above by

$$
\bar{c} \tau\left\|\varrho_{B}-r_{B}\right\|_{L^{1}\left(\Gamma_{\mathrm{in}}\right)}+\delta \int_{0}^{\tau}\|\mathbf{v}-\mathbf{V}\|_{W^{1,2}(\Omega)}^{2} \mathrm{~d} t+c^{\prime}(\delta) \int_{0}^{\tau} a(t) \mathcal{E}(\varrho, \mathbf{v} \mid r, \mathbf{V}) \mathrm{d} t
$$

with any $\delta>0$, where $c, \bar{c}>0$ are independent of $\delta, \bar{c}^{\prime} \geq 0, c^{\prime}=c^{\prime}(\delta)>0$, and $a \in L^{1}(0, T)$. This process leads to the estimate

$$
\mathcal{E}(\varrho, \mathbf{v} \mid r, \mathbf{V})(\tau) \leq \mathcal{E}\left(\varrho_{0}, \mathbf{v}_{0} \mid r(0), \mathbf{V}(0)\right)+\bar{c} T\left\|\varrho_{B}-r_{B}\right\|_{L^{1}\left(\Gamma_{\mathrm{in}}\right)}+c \int_{0}^{\tau} a(t) \mathcal{E}(\varrho, \mathbf{v} \mid r, \mathbf{V}) \mathrm{d} t
$$

that implies estimate (2.22) by the Gronwall inequality. In the rest of this section, we shall perform this program.

We start by observing that the bound from below (8.9) holds true with $\bar{c}^{\prime}=0$ and some $c=c(\mu)>0$. Indeed, to see this we may use the Korn type inequality

$$
\int_{\Omega} \mathbb{S}(\nabla \mathbf{w}): \nabla \mathbf{w} \quad \mathrm{d} x \geq \bar{c}\|\nabla \mathbf{w}\|_{L^{2}\left(\Omega ; \mathbb{R}^{3}\right)}^{2}
$$

holding for all $\mathbf{w} \in W_{0}^{1,2}\left(\Omega ; \mathbb{R}^{3}\right)$ with $\bar{c}>$ independent of $\mathbf{w}$ (which can be, in this setting, easily proved by using the integration by parts and a density argument) and the standard Poincaré inequality to deduce that

$$
c \int_{0}^{\tau}\|\mathbf{v}-\mathbf{V}\|_{W^{1,2}\left(\Omega ; \mathbb{R}^{3}\right)}^{2} \mathrm{~d} t+\left.\mathcal{E}(\varrho, \mathbf{v} \mid r, \mathbf{V})\right|_{0} ^{\tau} \leq \int_{0}^{\tau} \int_{\Omega} \mathbb{S}\left(\nabla(\mathbf{v}-\mathbf{V}): \nabla(\mathbf{v}-\mathbf{V}) \mathrm{d} x \mathrm{~d} t+\left.\mathcal{E}(\varrho, \mathbf{v} \mid r, \mathbf{V})\right|_{0} ^{\tau}\right.
$$

\subsection{Estimates of the remainder}

We introduce essential and residual sets in $\Omega$. To this end we take in (8.7) $a=\underline{r}, b=\bar{r}$ and define for a.e. $t \in(0, T)$ the residual and essential subsets of $\Omega$ as follows:

$$
N_{\text {ess }}(t)=\left\{x \in \Omega \mid \varrho(t) \in \mathcal{O}_{\text {ess }}\right\}, N_{\text {res }}(t)=\Omega \backslash N_{\text {ess }}(t) .
$$

With this definition at hand and having assumption (2.20) in mind, we deduce from Lemma 8.2 and Corollary 8.3

$$
c \int_{\Omega}\left([1]_{\mathrm{res}}+[\varrho]_{\mathrm{res}}+[p(\varrho)]_{\mathrm{res}}+[\varrho-r]_{\mathrm{ess}}^{2}\right) \mathrm{d} x \leq \int_{\Omega} E(\varrho, \mathbf{u} \mid r, \mathbf{U}) \mathrm{d} x
$$


with some $c=c(\underline{r}, \bar{r})>0$, where we denote, for a function $h$ defined a.e. in $(0, T) \times \Omega$,

$$
[h]_{\mathrm{ess}}=h 1_{N_{\mathrm{ess}}},[h]_{\mathrm{res}}=h 1_{N_{\mathrm{res}}} .
$$

We are now in position to estimate the remainder $\mathcal{R}$ at right hand side of the relative energy inequality (8.2). We shall do it in five steps.

Step 1: The surface integral in $\mathcal{R}$

We have immediately by the Taylor formula

$\left.\mid \int_{0}^{\tau} \int_{\Gamma_{\mathrm{in}}}\left(H\left(r_{B}\right)-H\left(\varrho_{B}\right)+\left(\varrho_{B}-r_{B}\right) H^{\prime}\left(r_{B}\right)\right) \mathbf{u}_{B} \cdot \mathbf{n}\right) \mathrm{d} S \mathrm{~d} t \mid \leq T c\left(\left|H^{\prime}\right|_{C([\mathfrak{r}, \overline{\mathrm{r}}])},\left|\mathbf{u}_{B}\right| C(\partial \Omega)\right)\left\|\varrho_{B}-r_{B}\right\|_{L^{1}\left(\Gamma_{\mathrm{in}}\right)}$.

Step 2: The first volume integral in $\mathcal{R}$

We shall first estimate the "essential part" of the first two terms:

$$
\begin{aligned}
& \int_{0}^{\tau} \int_{\Omega}[1]_{\mathrm{ess}}(\varrho-r)\left(\partial_{t} \mathbf{U}+\mathbf{U} \cdot \nabla_{x} \mathbf{U}\right) \cdot(\mathbf{V}-\mathbf{v}) \mathrm{d} x \mathrm{~d} t \\
& \quad \leq \int_{0}^{\tau}\left\|\partial_{t} \mathbf{U}+\mathbf{U} \cdot \nabla_{x} \mathbf{U}\right\|_{L^{\infty}\left(\Omega ; \mathbb{R}^{3}\right)}\left\|[\rho-r]_{\mathrm{ess}}\right\|_{L^{2}(\Omega)}\|\mathbf{v}-\mathbf{V}\|_{L^{2}\left(\Omega ; R^{3}\right)} \mathrm{d} t \\
& \quad \leq \delta \int_{0}^{\tau}\|\mathbf{v}-\mathbf{V}\|_{L^{2}\left(\Omega ; \mathbb{R}^{3}\right)}^{2} \mathrm{~d} t+c(\delta, \underline{r}, \bar{r}) \int_{0}^{\tau} a(t) \mathcal{E}(\varrho, \mathbf{v} \mid r, \mathbf{V}) \mathrm{d} t
\end{aligned}
$$

where

$$
a=\left\|\partial_{t} \mathbf{U}+\mathbf{U} \cdot \nabla_{x} \mathbf{U}\right\|_{L^{\infty}\left(\Omega ; \mathbb{R}^{3}\right)}^{2} \in L^{1}(0, T) .
$$

Concerning the "residual part", we shall estimate the integrals over the sets $\{\varrho \leq \underline{r} / 2\}$ and $\{\varrho \geq 2 \bar{r}\}$ separately.

$$
\begin{aligned}
& \int_{0}^{\tau} \int_{\Omega} 1_{\{\varrho \leq \underline{r} / 2\}}(\rho-r)\left(\partial_{t} \mathbf{U}+\mathbf{U} \cdot \nabla_{x} \mathbf{U}\right) \cdot(\mathbf{V}-\mathbf{v}) \mathrm{d} x \mathrm{~d} t \\
& \quad \leq 2 \bar{r} \int_{0}^{\tau} \int_{\Omega} 1_{\text {res }}\left|\partial_{t} \mathbf{U}+\mathbf{U} \cdot \nabla_{x} \mathbf{U}\right||\mathbf{V}-\mathbf{v}| \mathrm{d} x \mathrm{~d} t \\
& \quad \leq 2 \bar{r} \int_{0}^{\tau}\left\|\partial_{t} \mathbf{U}+\mathbf{U} \cdot \nabla_{x} \mathbf{U}\right\|_{L^{\infty}\left(\Omega ; \mathbb{R}^{3}\right)}\left\|1_{\mathrm{res}}\right\|_{L^{2}(\Omega)}\|\mathbf{v}-\mathbf{V}\|_{L^{2}\left(\Omega ; \mathbb{R}^{3}\right)} \mathrm{d} t \\
& \quad \leq \delta \int_{0}^{\tau}\|\mathbf{v}-\mathbf{V}\|_{L^{2}\left(\Omega ; \mathbb{R}^{3}\right)}^{2} \mathrm{~d} t+c(\delta, \underline{r}, \bar{r}) \int_{0}^{\tau} a(t) \mathcal{E}(\varrho, \mathbf{v} \mid r, \mathbf{V}) \mathrm{d} t
\end{aligned}
$$

where $a$ is given in (8.16).

Finally,

$$
\int_{0}^{\tau} \int_{\Omega} 1_{\{\varrho \geq 2 \bar{r}\}}(\varrho)(\varrho-r)\left(\partial_{t} \mathbf{U}+\mathbf{U} \cdot \nabla_{x} \mathbf{U}\right) \cdot(\mathbf{V}-\mathbf{v}) \mathrm{d} x \mathrm{~d} t
$$




$$
\begin{aligned}
& \leq 2 \int_{0}^{\tau} \int_{\Omega}[1]_{\mathrm{res}} \sqrt{\varrho}\left|\partial_{t} \mathbf{U}+\mathbf{U} \cdot \nabla_{x} \mathbf{U}\right| \sqrt{\varrho}|\mathbf{V}-\mathbf{v}| \mathrm{d} x \mathrm{~d} t \\
& \leq \int_{0}^{\tau}\left\|\partial_{t} \mathbf{U}+\mathbf{U} \cdot \nabla_{x} \mathbf{U}\right\|_{L^{\infty}\left(\Omega ; \mathbb{R}^{3}\right)}\left\|[\varrho]_{\mathrm{res}}\right\|_{L^{1}(\Omega)}^{1 / 2}\left\|\varrho(\mathbf{v}-\mathbf{V})^{2}\right\|_{L^{1}(\Omega)}^{1 / 2} \mathrm{~d} t \\
& \leq c(\underline{r}, \bar{r}) \int_{0}^{\tau} a(t) \mathcal{E}(\varrho, \mathbf{v} \mid r, \mathbf{V}) \mathrm{d} t
\end{aligned}
$$

with the same $a$ as before. In all above three formulas, we have employed (8.14) in the passage to their last lines.

As far as the third term is concerned, we have immediately,

$$
\int_{0}^{\tau} \int_{\Omega} \varrho(\mathbf{v}-\mathbf{V}) \cdot \nabla_{x} \mathbf{U} \cdot(\mathbf{V}-\mathbf{v}) \mathrm{d} x \mathrm{~d} t \leq c \int_{0}^{\tau} a(t) \mathcal{E}(\varrho, \mathbf{v} \mid r, \mathbf{V}) \mathrm{d} t
$$

with

$$
a=\|\nabla \mathbf{U}\|_{L^{\infty}\left(\Omega ; R^{9}\right)} \in L^{2}(0, T) .
$$

Resuming, the first volume integral in the remainder $\mathcal{R}$ of $(8.2)$ is bounded from above by

$$
\delta \int_{0}^{\tau}\|\mathbf{v}-\mathbf{V}\|_{W^{1,2}\left(\Omega ; \mathbb{R}^{3}\right)}^{2} \mathrm{~d} t+c \int_{0}^{\tau} a(t) \mathcal{E}(\varrho, \mathbf{v} \mid r, \mathbf{V}) \mathrm{d} t
$$

where $\delta>0, c=c(\delta, \underline{r}, \bar{r})>0$ and

$$
a=\|\nabla \mathbf{U}\|_{L^{\infty}\left(\Omega ; \mathbb{R}^{9}\right)}+\left\|\partial_{t} \mathbf{U}+\mathbf{U} \cdot \nabla_{x} \mathbf{U}\right\|_{L^{\infty}\left(\Omega ; \mathbb{R}^{3}\right)}^{2} \in L^{1}(0, T) .
$$

Step 3: The second volume integral in $\mathcal{R}$

As far as the last term is concerned, we use: 1) The Taylor formula together with the regularity $C^{2}$ of the pressure $p$, in order to estimate the essential part

$$
\begin{aligned}
& -\int_{0}^{\tau} \int_{\Omega}\left[p(\rho)-p^{\prime}(r)(\rho-r)-p(r)\right]_{\mathrm{ess}} \operatorname{div} \mathbf{U} \mathrm{d} x \mathrm{~d} t \\
& \quad \leq c\left(\underline{r}, \bar{r},\left|p^{\prime}\right|_{C^{1}([\underline{r} / 2,2 \bar{r}])}\right) \int_{0}^{\tau}\|\operatorname{div} \mathbf{U}\|_{L^{\infty}(\Omega)}\left\|[\varrho-r]_{\mathrm{ess}}\right\|_{L^{2}(\Omega)}^{2} \\
& \quad \leq c\left(\underline{r}, \bar{r},\left|p^{\prime}\right|_{C^{1}([\underline{[} / 2,2 \bar{r}])}\right) \int_{0}^{\tau} a(t) \mathcal{E}(\varrho, \mathbf{v} \mid r, \mathbf{V}) \mathrm{d} t, \quad a=\|\operatorname{div} \mathbf{U}\|_{L^{\infty}(\Omega)} \in L^{2}(0, T) .
\end{aligned}
$$

2) Employing Lemma 8.2, hypotheses (2.20) and Corollary 8.3, we deduce the pointwise bound,

$$
\left|\left[p(\rho)-p^{\prime}(r)(\rho-r)-p(r)\right]_{\mathrm{res}}\right| \leq c\left(\underline{r}, \bar{r},|p|_{C^{1}([\underline{r}, \bar{r}])}\right) E(\varrho \mid r)
$$

in order to estimate the residual part

$$
-\int_{0}^{\tau} \int_{\Omega}\left[p(\rho)-p^{\prime}(r)(\rho-r)-p(r)\right]_{\mathrm{res}} \operatorname{div} \mathbf{U} \mathrm{d} x \mathrm{~d} t \leq c\left(\underline{r}, \bar{r},|p|_{C^{1}([\underline{r}, \bar{r}])}\right) \int_{0}^{\tau} a(t) \mathcal{E}(\varrho, \mathbf{v} \mid r, \mathbf{V}) \mathrm{d} t
$$


We resume estimates obtained in Step 2:

$$
-\int_{0}^{\tau} \int_{\Omega}\left[p(\rho)-p^{\prime}(r)(\rho-r)-p(r)\right]_{\mathrm{ess}} \operatorname{div} \mathbf{U} \mathrm{d} x \mathrm{~d} t \leq c \int_{0}^{\tau} a(t) \mathcal{E}(\varrho, \mathbf{u} \mid r, \mathbf{V}) \mathrm{d} t
$$

where

$$
a=\|\operatorname{div} \mathbf{U}\|_{L^{\infty}(\Omega)} \in L^{2}(0, T), c=c\left(\underline{r}, \bar{r},|p|_{C^{2}([\underline{r} / 2,2 \bar{r}])}\right)>0 .
$$

Step 4: The third volume integral in $\mathcal{R}$

Similarly as in Step 1,

$$
\begin{aligned}
& \int_{0}^{\tau} \int_{\Omega} \frac{\nabla_{x} p(r)}{r}(r-\rho) \cdot(\mathbf{v}-\mathbf{V}) \mathrm{d} x \mathrm{~d} t \\
& \quad \leq \delta \int_{0}^{\tau}\|\mathbf{v}-\mathbf{V}\|_{W^{1,2}\left(\Omega ; \mathbb{R}^{3}\right)}^{2} \mathrm{~d} t+c(\delta, \underline{r}, \bar{r}) \int_{0}^{\tau} a(t) \mathcal{E}(\varrho, \mathbf{v} \mid r, \mathbf{V}) \mathrm{d} t
\end{aligned}
$$

where

$$
a=\left\|\frac{\nabla_{x} p(r)}{r}\right\|_{L^{\infty}\left(\Omega ; \mathbb{R}^{3}\right)}^{2} \in L^{1}(0, T) .
$$

Step 5:Conclusion

Coming back with these estimates to the relative energy inequality (8.2), taking into account (8.12) and choosing $\delta$ sufficiently small with respect to $\mu$, we easily verify the validity of (8.11). This finishes the proof of Theorem 2.4 .

\section{Concluding remarks}

\subsection{Existence of dissipative solutions in domains with piecewise regular boundaries}

So far we have established the existence of dissipative weak solutions under the assumption of the $C^{2}$ regularity of the domain. In many practical situations in nonzero outflow/inflow regimes, the domain occupied by the fluid does not possess this regularity. A typical example of such situation is a finite cylinder with inflow and outflow boundaries lower and upper discs of the boundary of the cylinder. The present section intends to remove this drawback.

We start with the definition of the piecewise $C^{2}$ Lipschitz domain.

Definition 9.1[Piecewise $C^{2}$ Lipschitz domain]

We shall say that $\Omega \subset R^{d}, d=2,3$, is a bounded piecewise $C^{2}$ Lipschitz domain if

1. $\Omega$ is a bounded Lipschitz domain;

2. The boundary of the domain can be written as

$$
\partial \Omega=\Gamma \cup \gamma \quad \text { with } \Gamma=\cup_{i=1}^{I} \Gamma_{i}, \quad \gamma=\cup_{i=1}^{I} \gamma_{i}
$$


where $\Gamma_{i}$ are open connected $(d-1)$ dimensional mutually disjoint manifolds of class $C^{2}$ and

$$
\gamma_{i} \equiv \partial \Gamma_{i}=\cup_{k_{i}=1}^{K_{i}} \gamma_{i, k_{i}}
$$

When $d=3, \gamma_{i, k_{i}}$ is a closed parametrized curve in $R^{3}$ of class $C^{2} . \quad$ If $\gamma_{i, k_{i}}$ and $\gamma_{j, l_{j}}$ intersect, they coincide. When $d=2, \gamma_{i, k_{i}}$ is a point in $R^{2}$.

Theorem 9.1. Let $\varrho_{B}, \mathbf{u}_{B}, \varrho_{0}, \mathbf{u}_{0}$ and $p$ satisfy all assumptions of Theorem [2.2. We suppose that $\Omega$ is a piecewise $C^{2}$ Lipschitz domain such that:

1.

$$
\partial \Omega=\bar{\Gamma}_{0} \cup \bar{\Gamma}_{\text {in }} \cup \bar{\Gamma}_{\text {out }}
$$

2. There holds

$$
\begin{aligned}
& \Gamma_{\mathfrak{a}}=\cup_{i_{\mathfrak{a}}=1}^{I_{\mathfrak{a}}} \Gamma_{i_{\mathfrak{a}}} \\
& \bar{\Gamma}_{k_{\mathfrak{a}}} \cap \bar{\Gamma}_{l_{\mathfrak{b}}}=\emptyset \quad \text { where } \mathfrak{a} \text { stands for } \quad \text { whener } \mathfrak{a} \in\{\text { in, out }\}, \mathfrak{b} \in\{\text { in, out }\}, k_{\mathfrak{a}} \neq l_{\mathfrak{b}},
\end{aligned}
$$

where $\Gamma_{i_{\mathfrak{a}}}$ are (open, connected) $(d-1)$-dimensional mutually disjoint manifolds of class $C^{2}$, "in" and "out" refer to the notation (1.5) and

$$
\Gamma_{0}=\operatorname{int}_{d-1}\left(\left\{x \in \partial \Omega \mid \mathbf{u}_{B} \cdot \mathbf{n}=0\right\}\right) .
$$

In the above $\operatorname{int}_{d-1}$ the interior on the (hyper)surface $\partial \Omega$.

3. There holds

$$
\gamma_{\mathfrak{a}} \equiv \partial \Gamma_{\mathfrak{a}}=\cup_{k_{\mathfrak{a}}=1}^{K_{\mathfrak{a}}} \gamma_{\mathfrak{a}, k_{\mathfrak{a}}}
$$

where $\gamma_{\mathfrak{a}, k_{\mathfrak{a}}}$ is a closed parametrized curve in $R^{d}$ of class $C^{2}$ (if $d=3$ ) or a point (if $d=2$ ) such that either $\gamma_{\mathfrak{a}, k_{\mathfrak{a}}} \cap \gamma_{\mathfrak{b}, l_{\mathfrak{b}}}=\emptyset$ or $\gamma_{\mathfrak{a}, k_{\mathfrak{a}}}=\gamma_{\mathfrak{b}, l_{\mathfrak{b}}}$.

Then all conclusions of Theorem 2.2 remain valid, in particular, the problem (1.1]1.5) admits a dissipative weak solution.

Remark 9.2. 1. The main issue of the proof of Theorem 9.1 is a construction of a convenient approximation of the piecewise regular domain $\Omega$ by regular $\left(C^{2}\right)$ bounded domains $\Omega_{\kappa}$ (small parameter $\kappa>0$ ) keeping conserved up to small perturbations the inflow/outflow properties of the fluid flow. Such approximation of the domain and boundary data has been suggested in [5, Section 3] and existence of bounded energy weak solutions has been proved through the limit passage $\kappa \rightarrow 0$ from bounded energy weak solutions on $C^{2}$-domains $\Omega_{\kappa}$ to bounded energy weak solutions on domain $\Omega$. Likewise, using ideas of Section 6, one can pass to the limit also from the relative energy inequality on $\Omega_{\kappa}$ to relative energy inequality on $\Omega$. We let the details to the interested reader.

2. The dissipative weak solutions constructed in Theorem 9.1 fulfill all assumptions of Theorem 2.4. In particular, they satisfy the weak-strong uniqueness principle. 


\subsection{Nonmonotone pressure law}

The statement of Theorem 2.2 and also of Theorem 9.1 can be generalized to some possibly non monotone pressure laws, as, e.g.,

$$
\begin{gathered}
p=\pi+\mathfrak{p}, \pi \in C[0, \infty) \cap C^{1}(0, \infty), \pi(0)=0, \pi^{\prime}(\varrho)>0, \\
\pi^{\prime}(\varrho) \geq a_{1} \varrho^{\gamma-1}-b, \pi(\varrho) \leq a_{2} \varrho^{\gamma}+b
\end{gathered}
$$

with $\gamma>d / 2$ and $a_{1}, a_{2}>0, b \geq 0$, and

$$
\mathfrak{p} \in C_{c}^{2}[0, \infty), \mathfrak{p} \leq 0
$$

In this case the existence of bounded energy weak solutions in non zero inflow/outflow setting has been proved in [3] and [5] and consequently the construction of dissipative weak solution can be performed by pursuing the strategy of the present paper.

Likewise the statement of Theorem 2.4 can be generalized to the pressure laws (9.5) with $\gamma>1$ and with

$$
\mathfrak{p} \in C_{c}^{2}[0, \infty) \text { globally Lipschitz. }
$$

In this case the relative energy function $E(\varrho \mid r)$ defined in (2.4) must be calculated from the monotone part of the pressure, i.e., with

$$
H(\varrho)=\varrho \int_{1}^{\varrho} \frac{\pi(z)}{z^{2}} \mathrm{~d} z .
$$

This result can be proved by combining the strategy of the present paper with the ideas introduced in Feireisl [12] and Chaudhuri [4].

\section{References}

[1] D. Bresch And P.-E. JABIn, Global existence of weak solutions for compresssible Navier-Stokes equations: Thermodynamically unstable pressure and anisotropic viscous stress tensor, Ann. of Math., 188 (2018), pp.-577-684.

[2] S. Benzoni-Gavage, Calcul différentiel et équations différentielles, Dunod, 2010.

[3] T. Chang, B.J. Jin, A. Novotny Compressible Navier-Stokes system with general inflow-outflow boundary data SIAM J. Math. Anal., 2019, accepted

[4] N. Chaudhuri On weak-strong uniqueness for compressible Nvier-Stokes system with general pressure laws NORWA, 2019, accepted

[5] H.J. Choe, A. Novotny, M. YAng Compressible Navier-Stokes system with general inflowoutflow boundary data on piecewise regular domains ZAMM Z. Angew. Math. Mech. 98(8) (2018), pp. 1447-1471 
[6] C.M. Dafermos. The second law of thermodynamics and stability. Arch. Rational Mech. Anal. 70 (1979), pp. 167-179

[7] R. Denk, M. Hieber, And J. Prüss, Optimal Lp-Lq-estimates for parabolic boundary value problems with inhomogeneous data, Math. Z., 257(2007), pp.-193-224.

[8] R.J. DiPerna And P.-L. Lions, Ordinary differential equations, transport theory and Sobolev spaces, Invent. Math., 98(1989), pp.-511-547.

[9] L. C. Evans, Partial Differential Equations, Graduate Studies in Mathematics, Vol. 19, AMS

[10] E. FeIREIsL, Dynamics of viscous compressible fluids, Oxford University Press, Oxford, 2004.

[11] E. FEIREISL, Compressible Navier-Stokes equations with a non-monotone pressure law, J. Differential Equations, 184 (2002), pp.-97-108.

[12] E. FEIREISL, On weak-strong uniqueness for the compressible Navier-Stokessystem with non-monotone pressure law, Preprint 27-2018, Math. Inst. Czech. Acad. Sci, http://www.math.cas.cz/fichier/preprints/IM_20180623094424_14.pdf

[13] E. Feireisl, B J. Jin, And A. Novotný Relative entropies, suitable weak solutions, and weakstrong uniqueness for the compressible Navier-Stokes system. J. Math. Fluid. Mech., 14(4):717-730, 2012.

[14] E. Feireisl and A. Novotný, Singular limits in thermodynamics of viscous fluids, Birkhauser, Basel, 2009. 2nd. enlarged edition 2017

[15] E. Feireisl, A. Novotný And H. Petzeltová, On the existence of globally defined weak solutions to the Navier-Stokes equations of compressible isentropic fluids, J. Math. Fluid Mech., 3(2001), 358-392.

[16] E. Feireisl, A. Novotný, Y. Sun Suitable weak solutions to the Navier-Stokes equations of compressible viscous fluids Indiana Univ. Math. J. 60, 2011, 611 - 632

[17] T. Gallouet, R. Herbin, D. Maltese, A. Novotny Error estimates for a numerical approximation to the compressible barotropic Navier-Stokes equations, IMA Journal of Numerical Analysis, 36(2) (2016), pp. 543-592

[18] T. Gallouet, D. Maltese, A. Novotny Error estimates for the implicit MAC scheme for the compressible NavierStokes equations., Numer. Math. 141(2), 2019, 495-567

[19] R. Klein, N. Botta, T. Schneider, C.D. Munz, S. Roller, A. Meister, L. Hoffman, T. SonAR Asymptotic adaptive methods for multi-scle problems in fluid mechanics. J. Eng. Math. 39 (2001), pp. 537-559 
[20] G. P. GALDI An introduction to the mathematical theory of the Navier-Stokes equations I. Springer, New-York, Second edition, 2011

[21] V. GiRinon, Navier-Stokes equations with nonhomogeneous boundary conditions in a bounded three-dimensional domain, J. Math. Fluid Mech., 13(2011), pp.-309-339.

[22] P.-L. Lions, Mathematical topics in fluid dynamics, Vol.2, Compressible models, Oxford Science Publication, Oxford, 1998.

[23] S. Novo, Compressible Navier-Stokes model with inflow-outflow boundary conditions, J. Math. Fluid Mech., 7(2005), pp.-485-514.

[24] A. Novotný And I. StrašKraba, Introduction to the mathematical theory of compressible flow, Oxford University Press, Oxford, 2004.

[25] A. Valli and M. ZaJaczKowski, Navier-Stokes equations for compressible fluids: Global existence and qualitative properties of the solutions in the general case, Comm. Math. Phys., 103(1986), pp.-259-296, 1986. 